\title{
Bio-based composite hydrogels for biomedical applications
}

\author{
Sytze J. Buwalda
}

MINES ParisTech, PSL Research University, Center for Materials Forming (CEMEF), UMR

CNRS 7635, CS 10207, 06904 Sophia Antipolis, France

\begin{abstract}
$\underline{\text { Abstract }}$
Hydrogels are three-dimensional, water-swollen polymer networks that have been widely studied for biomedical applications such as tissue engineering and the controlled delivery of biologically active agents. Since the pioneering work of Wichterle and Lim in the 1960s, hydrogel research has shifted from relatively simple single polymer networks to multifunctional composite hydrogels that better mimic the complex nature of living tissues. Bio-based polymers, which can be obtained from renewable natural resources, attract increasing attention for use in biomaterials in view of the recent demands for a reduction in the environmental impact of the polymer industry and the development of a sustainable society. Moreover, biobased polymers are often biodegradable and exhibit a significant level of biocompatibility and biomimicry, which are highly desired properties with regard to in vivo application. This review presents the state-of-the-art in the field of bio-based composite hydrogels for biomedical applications, thereby focusing on different types of polymeric components that have been combined with hydrogels to obtain materials with unique, synergistic properties: particles (including micelles and microspheres), electrospun fibres and nanocellulose. In addition, the challenges are described that should be overcome to facilitate clinical application of these versatile and environmentally responsible biomaterials.
\end{abstract}




\section{Keywords}

Composites; bio-based polymers; hydrogels; fibers; particles; biomedical.

\section{Contents}

1. Introduction

2. Bio-based polymers as building blocks for composite hydrogels

3. Bio-based composites of polymer particles and hydrogels

4. Bio-based composites of electrospun fibers and hydrogels

5. Bio-based composites of nanocellulose and hydrogels

6. Conclusions and outlook

7. References

\section{Introduction}

Hydrogels are polymeric networks based on hydrophilic macromonomers that are able to retain large amounts of water. ${ }^{1-5}$ They generally exhibit excellent biocompatibility and their mechanical properties can be designed in such a way that they match those of many biological tissues. Furthermore, their soft and rubbery nature minimizes inflammatory reactions of the surrounding cells and tissues. Hydrogels are consequently used for a wide range of biomedical applications, including controlled drug delivery and tissue engineering. They can be physically crosslinked by noncovalent interactions, chemically crosslinked by covalent bonds, or crosslinked by a combination of both.

Hydrogels may be classified as natural or synthetic, depending on the source of the constituting polymers. Synthetic polymers allow for a high control over the macromonomer structure and properties, but their synthesis often relies on petroleum-derived chemistry, refinery, and engineering processes which may have a negative impact on the environment. ${ }^{6}$ In particular, 
since the late 1990s, the polymer industry has faced two serious problems: global warming and depletion of fossil resources. One solution in addressing these problems is to use sustainable resources instead of fossil-based resources. Biomass feedstock can be converted into raw materials for polymer production, and the resulting polymers are called bio-based polymers. Natural polymers formed by plants, microorganisms, and animals constitute an important class of bio-based polymers. ${ }^{7}$ Such naturally derived biomass polymers offer great promise for reducing the environmental impact of the polymer industry and for realizing a bio-based and sustainable society. In addition to the environmental benefits, bio-based polymers also offer a high level of biocompatibility and biomimicry, thereby providing biophysical and biochemical properties which are able to induce the correct biological response for both in vitro and in vivo biomedical applications. ${ }^{8}{ }^{9}$ Moreover, these natural macromolecules are biodegradable and many of them also possess intrinsic antibacterial and anti-inflammatory properties. The present review focuses on bio-based hydrophilic polymers, including naturally derived biomass polymers that have been slightly chemically modified, such as methylcellulose.

Composite materials consist of two or more constituent materials with markedly different physicochemical properties. ${ }^{10}$ When the components are combined in a single entity where the individual components remain distinct and separate, the resultant composite material can exhibit different characteristics from the individual components and offer synergistic properties. Importantly, composite materials may also perform several tasks via ingenious combinations of multiple functional capabilities. ${ }^{11}$ Early hydrogels were mostly based on a single (co)polymer and often designed to perform only one task. ${ }^{2}$ In the past few decades, hydrogel research has focused increasingly on composite hydrogels which offer more opportunities to mimic the composite nature of biological tissues.

This review discusses recent approaches aimed at producing composite bio-based hydrogels for biomedical applications. First, an overview is given of the bio-based polymers that are treated 
in this review, followed by descriptions of the use of polymeric micro- or nanoparticles embedded in hydrogels, as well as the use of electrospun fibers in hydrogels to impart desired properties. Subsequently a separate chapter is dedicated to bio-based hydrogels incorporating cellulose fibrous nanostructures with remarkable properties. Lastly, the author's vision on the future of this rapidly evolving area of research is presented.

\section{$\underline{\text { 2. Bio-based polymers as building blocks for composite hydrogels }}$}

Several bio-based polymers have been explored for the preparation of hydrogels and micro- or nano-sized functional structures such as particles and fibers. These polymers, generally polysaccharides or proteins, are derived from plants, microorganisms or animals and offer the advantages of biodegradability and inherent biocompatibility. Table 1 presents an overview of the most important polysaccharides and protein-based polymers that are addressed in this review. They will be briefly discussed below.

\section{$\underline{\text { Polysaccharides }}$}

Polysaccharides, consisting of monosaccharide units bound together by glycosidic linkages, are usually hydrophilic and therefore very suitable for the preparation of hydrogels. The most frequently used polysaccharides for preparation of the hydrogel component in bio-based composites are alginate, chitosan, gellan gum and hyaluronic acid (Table 1). Cellulose, the most abundant polymer on earth, has often been applied in the form of reinforcing (nano)fibers. 
Table 1. Overview of the most important polysaccharides and protein-based polymers that are discussed throughout this review.

\begin{tabular}{|c|c|c|c|c|}
\hline & Chemical structure & Natural resource & $\begin{array}{l}\text { Shape/state in } \\
\text { composite }\end{array}$ & Reference \\
\hline \multicolumn{5}{|l|}{ Polysaccharides } \\
\hline \multirow[t]{2}{*}{ Alginic acid } & & \multirow[t]{2}{*}{ Brown algae } & Hydrogel & $\begin{array}{l}55,58,62,69, \\
75,76,78,79, \\
85,92,100,101, \\
102,103,104, \\
107,108\end{array}$ \\
\hline & & & Microspheres & 54 \\
\hline \multirow[t]{5}{*}{ Cellulose } & & \multirow{5}{*}{$\begin{array}{l}\text { Green plants, natural } \\
\text { fibers, bacteria }\end{array}$} & Hydrogel & 84,99 \\
\hline & & & Fibers & 85 \\
\hline & & & Nanocrystals & $\begin{array}{l}95,97,98,100, \\
105\end{array}$ \\
\hline & & & Nanofibrils & $\begin{array}{l}92,96,99,101 \\
102,103,104 \\
106,107\end{array}$ \\
\hline & & & $\begin{array}{l}\text { Bacterial } \\
\text { nanocellulose }\end{array}$ & $\begin{array}{l}107,108,109, \\
110\end{array}$ \\
\hline \multirow[t]{3}{*}{ Chitosan } & & \multirow[t]{3}{*}{$\begin{array}{l}\text { Fungi, exoskeletons } \\
\text { of insects and } \\
\text { crustaceans }\end{array}$} & Hydrogel & $\begin{array}{l}52,54,62,63, \\
68,77,81,96, \\
97,98,105,109, \\
110\end{array}$ \\
\hline & & & Micelles & 55 \\
\hline & & & Microspheres & 63 \\
\hline \multirow[t]{2}{*}{ Gellan gum } & \multirow{2}{*}{ 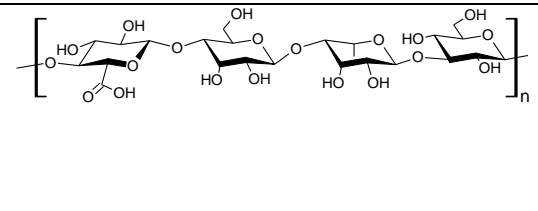 } & \multirow{2}{*}{$\begin{array}{l}\text { Bacteria, e.g. } \\
\text { Pseudomonas } \\
\text { elodea } \text { and } \\
\text { Sphingomonas } \\
\text { paucimobilis }\end{array}$} & Hydrogel & 51,95 \\
\hline & & & Micelles & 51 \\
\hline Hyaluronic acid & $\left.{ }_{\mathrm{NH}}\right]_{\mathrm{n}}$ & $\begin{array}{l}\text { Extracellular matrix } \\
\text { of soft connective } \\
\text { tissues }\end{array}$ & Hydrogel & $78,84,89,104$ \\
\hline \multicolumn{5}{|c|}{ Protein-based polymers } \\
\hline \multirow[t]{5}{*}{ Collagen/gelatin } & \multirow{5}{*}{$\begin{array}{l}\text { Type I collagen: triple-helical protein } \\
\text { of polypeptide chains (total MW } \\
\pm 300 \mathrm{kDa} \text { ) } \\
\text { Gelatin: hydrolytically degraded } \\
\text { collagen }\end{array}$} & \multirow[t]{5}{*}{ Connective tissues } & $\begin{array}{l}\text { Gelatin } \\
\text { hydrogel }\end{array}$ & $\begin{array}{l}80,82,85,100, \\
106\end{array}$ \\
\hline & & & $\begin{array}{l}\text { Gelatin } \\
\text { microspheres }\end{array}$ & 62,69 \\
\hline & & & Gelatin fibers & 75,76 \\
\hline & & & $\begin{array}{l}\text { Collagen } \\
\text { hydrogel }\end{array}$ & 83,108 \\
\hline & & & Collagen fibers & 79,84 \\
\hline \multirow[t]{2}{*}{ Silk fibroin } & \multirow{2}{*}{$\begin{array}{l}\text { Primary structure mainly consists of } \\
\text { repetitive motifs containing alanine, } \\
\text { glycine and serine amino acid } \\
\text { residues (total } \mathrm{MW} \pm 350 \mathrm{kDa} \text { ) }\end{array}$} & \multirow{2}{*}{$\begin{array}{l}\text { Cocoons or silk } \\
\text { glands of the } \\
\text { silkworm Bombyx } \\
\text { mori }\end{array}$} & Microparticles & 68 \\
\hline & & & Fibers & 81,89 \\
\hline
\end{tabular}


Alginate

Alginic acid is a polysaccharide that consists of $\alpha-1$-glucuronic acid $(\mathrm{G})$ and $\beta$-d-mannuronic acid (M) moieties. Its commercially available sodium salt, called sodium alginate, is typically obtained from brown seaweeds via treatment with aqueous alkali solutions, purification and further conversion. ${ }^{12}$ The properties of alginate depend largely on the sequence and ratio of $\mathrm{M}$ and $\mathrm{G}$ units, which in turn depends on the species of seaweed. A well-known method to prepare hydrogels from aqueous alginate solutions is the addition of divalent cations (most commonly $\mathrm{Ca}^{2+}$ ), which bind only to $\mathrm{G}$ units, as their structure allows a high degree of coordination with divalent ions. The $\mathrm{G}$ blocks of one alginate chain then form crosslinks with the $\mathrm{G}$ blocks on adjacent polymer chains in the so-called 'egg-box' model of crosslinking, resulting in a hydrogel. This mild preparation method makes these hydrogels suitable for encapsulation of living cells and for the controlled release of fragile biomolecules. ${ }^{13},{ }^{14}$ However, since the properties of alginate hydrogels depend mainly on the type and concentration of the used alginate, the possibilities to control, for example, the release behavior from the hydrogels are limited. $^{15}$

\section{Cellulose}

Cellulose is a polymer of glucose and is found as the main constituent of plants and natural fibers including linen and cotton. ${ }^{16}$ The glucose units are linked by $1,4-\beta$-glycosidic linkages, which account for the high crystallinity of native cellulose and its insolubility in water and other common solvents. Hydrogels can be prepared by dissolving native cellulose in solvents such as $\mathrm{N}$-methylmorpholine-N-oxide monohydrate or ionic liquids, followed by coagulation of the cellulose with water. ${ }^{17}$ It should be noted that because water is a non-solvent for cellulose, the term 'hydrogel' is, strictly speaking, not correct. Using a crosslinker such as epichlorohydrin, it is also possible to obtain chemically crosslinked hydrogels from native cellulose dissolved in 
alkali aqueous systems. ${ }^{18}$ Chemical modification of cellulose, usually via esterification or etherification of the hydroxyl groups, is performed to produce cellulose derivatives, which exhibit a better processability as well as a higher solubility in water. Cellulose-based hydrogels can therefore also be formed by properly crosslinking aqueous solutions of such cellulose derivatives, including methyl cellulose, hydroxypropyl cellulose, hydroxypropyl methyl cellulose and carboxymethyl cellulose. Advantages of cellulose hydrogels include biodegradability, biocompatibility, transparency and low costs. ${ }^{19,} 20$

Thanks to their wide availability, environmental friendliness and impressive physicomechanical properties, cellulose nanofibers have been frequently incorporated in hydrogels to add mechanical strength and other desired properties to the final composite system. ${ }^{21,22}$ Composite hydrogels containing nano-sized cellulose fibers, including nanocrystals and nanofibrils, will be discussed in the last section of this review.

\section{Chitosan}

Chitosan is a derivative of chitin, a main constituent of the cell walls of fungi and the exoskeletons of insects as well as crustaceans. Chitin is a linear polysaccharide comprised of $\beta-1,4-\mathrm{N}$-acetylglucosamine. It is insoluble in most solvents because of its high crystallinity and hydrogen bonding between carbonyl, hydroxyl and acetamide groups. To increase its processability, chitin is deacetylated to form chitosan, which is composed of glucosamine and $\mathrm{N}$-acetylglucosamine units. ${ }^{23}$ The cationic polymer chitosan is soluble in dilute acidic solutions and forms a hydrogel at higher $\mathrm{pH}$, resulting from the neutralization of amino groups and the subsequent removal of interchain electrostatic forces, which subsequently enables the formation of hydrophobic interactions and hydrogen bonding between neighbouring chains. ${ }^{24}$ The amino groups also facilitate chemical crosslinking of chitosan chains, for example via Schiff base formation using aldehyde-containing crosslinkers. ${ }^{25,26}$ The aqueous solubility of chitosan can 
be improved by carboxymethylation as a hydrophilic modification to yield carboxymethyl chitosan. ${ }^{27}$ Chitosan hydrogels have been extensively studied for various biomedical applications because of their antimicrobial activity, wound healing properties, haemostatic activity and mucoadhesiveness. ${ }^{28,} 29$ However, chitosan hydrogels often exhibit poor mechanical properties which limit their application.

\section{Gellan gum}

Gellan gum is a polysaccharide produced by certain bacteria, including Pseudomonas elodea and Sphingomonas paucimobilis. ${ }^{30}$ Deacetylated gellan gum consists of repeating tetrasaccharide units of D-glucose, D-glucuronic acid, D-glucose, and L-rhamnose. Gellan gum solutions can form physical hydrogels upon temperature decrease in the presence of cationic ions such as $\mathrm{Na}^{+}$and $\mathrm{Ca}^{2+}$. The gelation mechanism involves transition from a random coil to a double helix structure due to the temperature decrease followed by aggregation of the doublehelical segments via complexation with cations and hydrogen bonding with water. ${ }^{31}$ Advantages of gellan gum hydrogels include their transparency, $\mathrm{pH}$ stability and injectability. In view of the relatively high gel-sol transition temperature $\left(42.5^{\circ} \mathrm{C}\right)$ for in vivo injectability, Du et al. reduced the molecular weight of gellan gum chains via oxidative cleavage to bring the gelation point closer to body temperature. ${ }^{32}$

\section{Hyaluronic acid}

Hyaluronic acid is a non-sulphated glycosaminoglycan in the extracellular matrix of many soft connective tissues, composed of alternating units of D-glucuronic acid and N-acetyl-D glucosamine which are linked together via alternating $\beta-1,4$ and $\beta-1,3$ glycosidic bonds. Although aqueous hyaluronic acid solutions show viscoelasticity at high concentrations, such

physically crosslinked, entangled systems do not have long lasting mechanical integrity. ${ }^{33}$ For 
the preparation of hyaluronic acid hydrogels with controlled mechanical properties and degradation rates, chemical modification and covalent crosslinking are often necessary. As hyaluronic acid is non-immunogenic, non-thrombogenic and bioactive in terms of angiogenesis and dermal regeneration, its hydrogels have found widespread application as scaffolds for tissue engineering and regenerative medicine. ${ }^{34,35}$

\section{$\underline{\text { Protein-based polymers }}$}

Proteins are linear polymers of amino acids that have adopted a complex three-dimensional structure with several levels of structural organization. Especially structural proteins, including collagen and silk, have been exploited for the preparation of biomedical fibers ${ }^{36}$ and hydrogels. ${ }^{37}$

\section{Collagen and gelatin}

Collagen is the most abundant protein in mammals and comprises $25 \%$ (by dry weight) of the total body protein weight. Most collagen-based biomaterials are prepared using type I collagen, which constitutes $90 \%$ of the protein in human connective tissues and is easily extracted from animal tissue with minimal contamination by other collagens or proteins. ${ }^{38}$ Type I collagen is a triple-helical protein of polypeptide chains with a total molecular weight of approximately 300 $\mathrm{kDa}$. Collagen fibrils self-assemble at neutral $\mathrm{pH}$ in aqueous solution into bundled fibers that ultimately crosslink to form a physical hydrogel. Gelatin is derived from collagen by hydrolytic degradation. It is widely used in the food industry but also frequently employed as a material for biomedical applications. ${ }^{39,40}$ Since gelatin has a gel-sol transition temperature well below body temperature (around $30^{\circ} \mathrm{C}$ ), gelatin is often covalently crosslinked to avoid dissolution in vivo. ${ }^{8}$ Frequently the amine functional groups of gelatin are targeted via Schiff base reaction using glutaraldehyde. ${ }^{41}$ Collagen and gelatin hydrogels have been mainly investigated within 
the tissue engineering field and for the release of growth factors to promote tissue formation because of abundant favorable interactions between cells and these bio-based polymers. ${ }^{15}$ The fibrillar nature of natural collagen has inspired many researchers to mimic this element of its structure, which has been mainly achieved via electrospinning. ${ }^{42}$ Because of their excellent mechanical properties, electrospun collagen and gelatin fibers have been frequently used to reinforce bio-based hydrogels, as discussed in section 4 of this review.

Silk

Silk fibroin is a protein of approximately $350 \mathrm{kDa}$ that can be obtained in the form of fibers from the cocoons or silk glands of the silkworm Bombyx mori. Its primary structure mainly consists of repetitive motifs containing alanine, glycine and serine amino acid residues. These relatively hydrophobic blocks lead to abundant hydrogen bonding and hydrophobic interactions throughout the protein chains, resulting in a high crystallinity and environmental stability. ${ }^{43}$ The crystalline domains interact with dispersed, amorphous regions, providing silk biomaterials with exceptional strength as well as elasticity. ${ }^{44}$ Silk fibroin fibers also possess excellent processability and can been shaped into several forms including particles, ${ }^{45}$ electrospun mats ${ }^{46}$ and hydrogels. ${ }^{47}$ Silk-based biomaterials are highly compatible with various cell types and show a controlled degradation which is slower than that of many other bio-based polymers.

\section{$\underline{\text { 3. Bio-based composites of polymer particles and hydrogels }}$}

Hydrogels are attractive materials for the controlled release of drugs because of the possibility to incorporate therapeutic agents in their water-swollen network. Hydrogels have been used for localized and sustained release as a strategy to decrease the number of drug administrations, to protect the drug from degradation and to allow for therapeutic drug concentrations for prolonged times. ${ }^{1}$ Hydrogels were shown to be very useful for the controlled release of water- 
soluble compounds, but the hydrophilic nature of their matrices makes them unsuitable for the incorporation and release of hydrophobic drugs. ${ }^{48}$ Typically, such poorly water-soluble agents require encapsulation in micro- or nanosized particles to achieve controlled release in biological systems. This review will focus on two types of polymeric particles: micelles and microspheres. Micelles are nano-sized colloidal particles with a core-shell structure, which are spontaneously formed by the self-assembly of amphiphilic macromolecules in a block-selective solvent into nanoaggregates above a certain concentration, the critical micelle concentration. ${ }^{49}$ Microspheres are microparticles that consist of a solid and homogeneous polymer matrix. ${ }^{50}$ In order to obtain a controlled and sustained delivery of hydrophobic drugs from hydrogel-based devices, various researchers have followed the strategy to load the drugs in particles, followed by loading of these particles in the hydrogel matrix. For example, Kundu et al. reported on a micelle/hydrogel composite system based on gellan gum for oral drug delivery. ${ }^{51}$ By conjugating alkyl chains to the gellan gum backbone via an etherification reaction, amphiphilic copolymers were obtained. In aqueous solution these copolymers self-assembled into spherical micelles, which were able to encapsulate simvastatin, a hydrophobic drug used in the treatment of hypercholesterolemia. Composites were prepared by incorporating micelles in a native gellan gum solution, which was subsequently crosslinked by adding aluminium chloride resulting in ionic interactions between aluminium ions and the negatively charged gellan gum chains. Simvastatin-loaded native hydrogels exhibited a lower degree of swelling than the composite hydrogels containing simvastatin-loaded micelles, which was ascribed to the hydrophobicity of the dispersed drug in the monocomponent hydrogel. The in vitro simvastatin release was slower from native hydrogels than from composite hydrogels due to the combined effects of low hydrogel swelling and low drug solubility. The relatively fast release from the composites was explained by a mechanism in which the hydrogels swell, disintegrate and then release the drugloaded micelles. Following oral administration in rabbits, drug-loaded micelle/hydrogel 
composite beads were able to decrease cholesterol blood levels to a higher extent than drugloaded native hydrogel beads.

In addition to having cholesterol-lowering effects, simvastatin has been suggested as a potential agent to promote bone growth. Yan et al. prepared micelle/hydrogel composites for the controlled release of simvastatin to achieve local bone regeneration (Figure 1). ${ }^{52}$ Micelles were prepared from maltodextrin, a polysaccharide produced from starch by partial hydrolysis, whereas hydrogels were based on carboxymethyl chitosan. Esterification between maltodextrin and palmitic acid resulted in amphiphilic micelle-forming polymers, which were subsequently treated with an oxidizing agent in order to cleave a number of glycosidic bonds of the polymer backbone, yielding free aldehyde groups. Mixing aldehyde-functionalized micelles, aldehydefunctionalized maltodextrin and amine-bearing carboxymethyl chitosan resulted in the formation of a hydrogel network via Schiff base formation. As the micelles participated in the crosslinking reaction, they were anchored to the hydrogel network via chemical bonds. The gelation time could be tuned from 40 to 80 seconds by adjusting the degree of aldehyde functionalization of the micelles. Rheological measurements showed that the storage modulus of composites containing chemically anchored micelles was twice as high compared to control composites containing physically encapsulated micelles. Direct loading of simvastatin in the hydrogel resulted in phase separation between the hydrophobic drug and the matrix, as evidenced by a large number of visible drug aggregates and an opaque appearance (Figure 1). In contrast, hydrogels with simvastatin-loaded micelles showed good transparency, demonstrating the encapsulating effect of the micelles. The presence of chemical crosslinks between the micelles and the hydrogel network resulted in a more sustained release of micelles and their encapsulated simvastatin from the matrix compared to the control system with physically embedded micelles. In vitro experiments showed that the prolonged release of 
simvastatin from composites with chemically linked micelles facilitated the osteogenic differentiation of mouse osteoblast precursor cells.
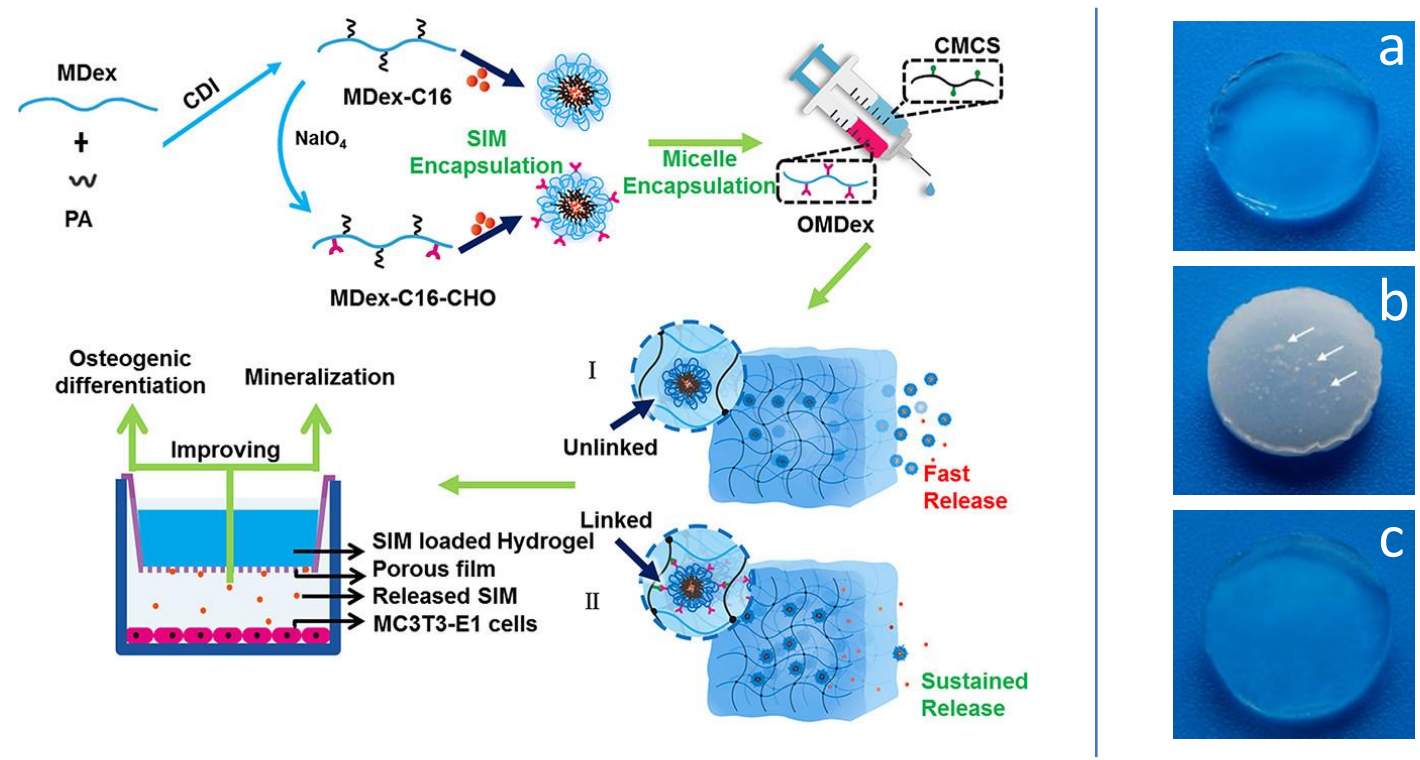

Figure 1. Left panel: Schematic illustration of the preparation of maltodextrin micelle/carboxymethyl chitosan hydrogel composites for the controlled release of simvastatin. Simvastatin-loaded aldehyde-modified micelles were linked to the hydrogel network via Schiff base formation, leading to sustained release of micelles and their encapsulated simvastatin. Osteoblast precursor cells were encapsulated in the composite hydrogels to demonstrate their cytocompatibility and osteogenic capability. Right panel: Images of blank hydrogel (a), simvastatin-loaded hydrogel (b, arrows indicate drug aggregates) and simvastatin-loaded micelle/hydrogel composite (c). Reprinted (adapted) with permission from reference 52. Copyright 2018 American Chemical Society.

A composite delivery system may also solve the issue of fast, uncontrolled dug release from particles alone. ${ }^{53}$ A more sustained overall drug release may be achieved via (1) release of drugloaded particles from the hydrogel and subsequent release of the drug from the particles or via (2) release of the drug from the entrapped particles and subsequent drug diffusion through the 
polymer matrix of the hydrogel. Which mechanism dominates will depend on various parameters such as the size of the hydrogel mesh and the particle, the hydrophilicity of the drug, as well as the chemical composition of the particle and hydrogel. Zhu et al. reported on a composite microsphere/hydrogel system for the controlled release of the hydrophilic drug berberine hydrochloride, which has anti-cancer, anti-inflammatory and antibiotic effetcs. ${ }^{54}$ The drug was loaded via an emulsification method into alginate microspheres, which were crosslinked with calcium ions. The drug-loaded microspheres $(10-15 \mu \mathrm{m}$ in size) were subsequently entrapped in a glutaraldehyde-crosslinked carboxymethyl chitosan hydrogel. Whereas the drug release into PBS ( $\mathrm{pH}$ 7.4) from the alginate microspheres or the chitosan hydrogel alone showed a large burst release, berberine hydrochloride was released at a nearly constant, zero-order rate from the composite system. When the $\mathrm{pH}$ of the release medium was decreased to 1.2, the release rate increased, which was ascribed to neutralization of the pendant $\mathrm{COO}^{-}$groups in the composite system, resulting in expulsion of the drug solution and hydrogel shrinkage. In addition to having advantageous effects on the drug release behaviour, the presence of microspheres increased the compressive strength of the hydrogels by $50 \%$.

Cong et al. reported on a $\mathrm{pH}$-responsive composite system consisting of chitosan micelles embedded in alginate hydrogel beads $(150 \mu \mathrm{m})$ for the oral delivery of the hydrophobic drug emodin, having antioxidant and antimicrobial activities, to the intestine. ${ }^{55}$ Micelles were prepared by mixing calcium chloride and chitosan solutions, leading to intrachain contraction of the cationic, amine-bearing chitosan chains by chloride anions and the formation of a crosslinked unimolecular micelle structure. Micelle-loaded hydrogels beads were obtained via dropwise addition of a micelle-containing sodium alginate solution into a calcium chloride solution. Transmission electron microscopy showed that the micellar diameter increased from $80 \mathrm{~nm}$ in aqueous solution to $100-200 \mathrm{~nm}$ when incorporated in hydrogel beads. The size increase was ascribed to the formation of a polyelectrolyte layer on the surface of the micelles. 
This layer was thought to originate from electrostatic interactions between carboxyl groups on alginate chains not participating in the hydrogel crosslinking and amino groups on chitosan chains not participating in the micellar crosslinking. Release experiments were performed by placing the micelles or micelle/hydrogel composites consecutively in simulated gastric fluid ( $\mathrm{pH} 1.2,2$ h), simulated small intestinal fluid $(\mathrm{pH} 6.8,3 \mathrm{~h}$ ) and simulated colon fluid ( $\mathrm{pH} 7.2$, $4 \mathrm{~h}$ ) to mimic the conditions in the gastro-intestinal tract. The emodin release from micelles alone was characterized by a burst release ( $80 \%$ release within $2 \mathrm{~h})$ due to dissociation of the micelles at acidic $\mathrm{pH}$ caused by strong electrostatic repulsion among the protonated amine groups. In sharp contrast, little burst release at $\mathrm{pH} 1.2(<20 \%$ for most formulations $)$ was observed when the micelles were incorporated in the alginate hydrogel beads. The protective effect of the hydrogel regarding micelle degradation and drug release was maintained when the composites were transferred to $\mathrm{pH} 6.8$ as the cumulative release increased only marginally during 3 h. At pH 7.4 however the cumulative release increased to $80 \%$ within 4 h. This was explained by degradation of the hydrogel caused by strong ionic repulsions between the alginate chains at $\mathrm{pH} 7.4$, leading to release of the micelles from the hydrogel and subsequent release of the drug from the micelles.

Composites of polymeric micro- or nanoparticles embedded in hydrogels may also facilitate the simultaneous delivery of multiple drugs with different solubility. This is important to enable combination therapy, which has shown several advantages compared to monotherapy such as reduced side effects ${ }^{56}$ and lowering of drug resistance. ${ }^{57}$ Zhong and co-workers prepared a dual drug delivery system by incorporating polylactide (PLA) microspheres in calcium crosslinked alginate hydrogel beads. ${ }^{58}$ PLA is a widely investigated biodegradable polyester that can be derived from renewable natural resources. ${ }^{59}$ The hydrophobic drug glycyrrhetinic acid (GA), having potential antiviral and antibacterial properties, was encapsulated in the PLA 
microspheres, whereas albumin was loaded in the hydrogel as a hydrophilic model protein drug. In this way, a more sustained GA release and a less significant burst were achieved compared to release from PLA microspheres alone. In vitro release experiments showed that albumin was released within 15 days, including an initial burst, whereas the delivery of GA was more sustained, with a release for multiple weeks. The release of albumin largely accelerated after increasing the PLA/alginate ratio, whereas at the same time, the release of GA decreased. Independent control over the individual drug releases, which is essential for successful combination therapy, was, however, not reported.

For biomedical applications, the use of in situ forming hydrogels is preferred over preformed hydrogels since there is no need for surgical interventions as gelation can take place under physiological conditions upon injection. Furthermore, the initial fluidic nature of the precursor solution ensures proper shape adaptation and biological components as well as particles can be incorporated in the hydrogel by simple mixing with the precursor polymer solution. ${ }^{60,61}$ Chen et al. designed an injectable composite hydrogel of gelatin microspheres in chitosan-alginate hydrogels for the delivery of the anti-cancer drug 5-fluorouracil (Figure 2). ${ }^{62}$ The hydrogel was crosslinked via Schiff base reaction between amino groups on carboxyethyl chitosan and aldehyde groups on oxidized alginate. The gelation time decreased from 150 to 75 seconds when drug-loaded gelatin microspheres were added to the hydrogel precursor solution, which was attributed to microsphere co-crosslinking via reaction of amino groups on the gelatin with aldehyde groups on the oxidized alginate. In addition to in situ gel formation, the composite hydrogel also showed self-healing behaviour, which was ascribed to the dynamic, reversible character of the Schiff base formation under selected conditions. The composite hydrogel showed a controlled release of 5-fluorouracil during 5 weeks, which was more sustained than the release from the hydrogel or the microspheres alone. Interestingly, incorporating magnetic 
$\mathrm{Fe}_{3} \mathrm{O}_{4}$ nanoparticles in the gelatin microspheres allowed for acceleration of the drug release by applying a magnetic field. The same group also reported on injectable hydrogels consisting of chitosan microspheres in carboxymethyl chitosan hydrogels for the release of albumin. ${ }^{63}$
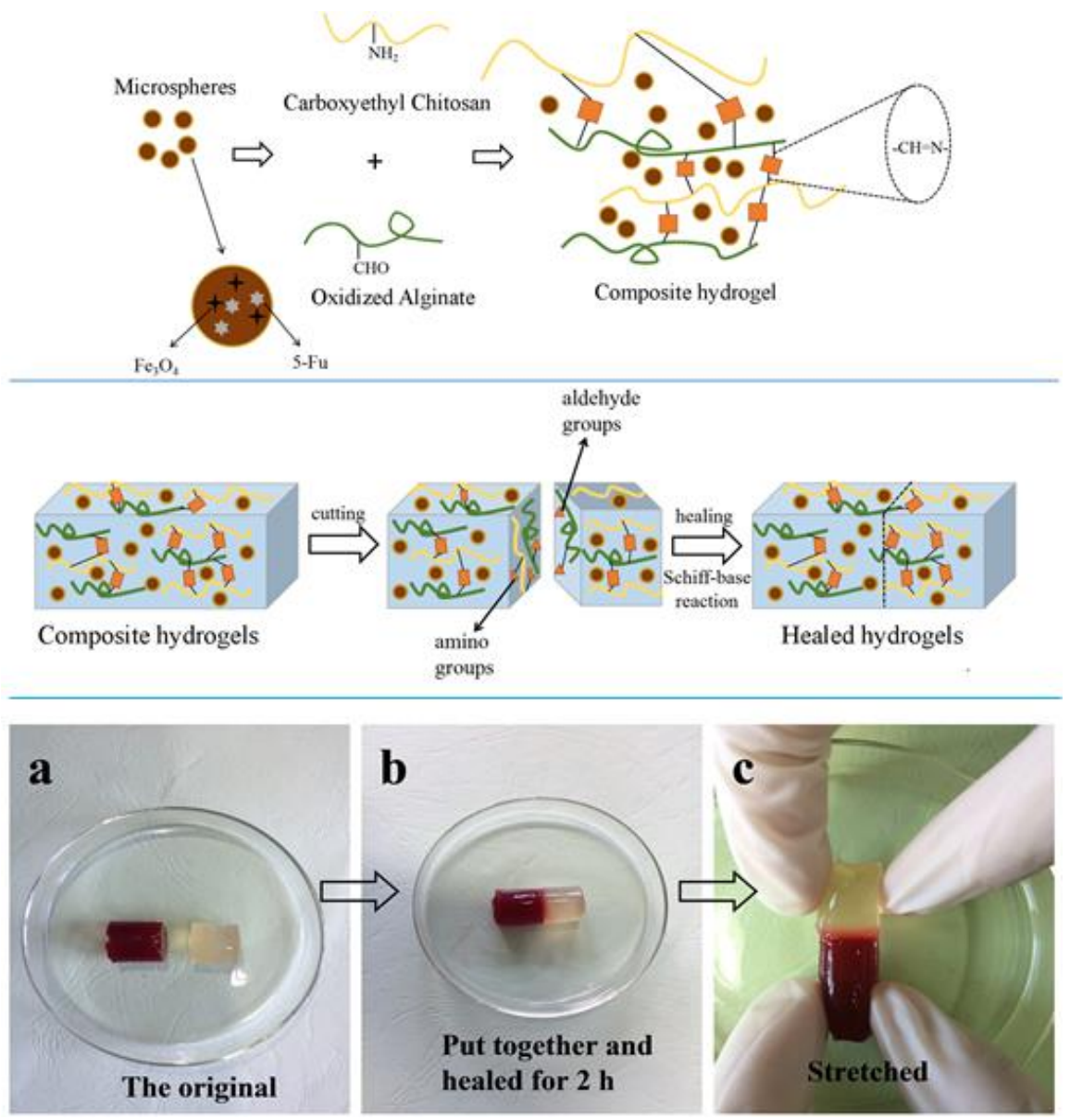

Figure 2. Top panel: Hydrogel crosslinking via Schiff base reaction between amino groups on carboxyethyl chitosan and aldehyde groups on oxidized alginate. Gelatin microspheres containing 5-fluorouracil and magnetic $\mathrm{Fe}_{3} \mathrm{O}_{4}$ nanoparticles were incorporated in the hydrogel precursor solutions. Middle panel: Schematic illustration of the self-healing capacity of the composite hydrogel. Bottom panel: Self-healing process of the blank chitosan-alginate hydrogel. Reprinted from reference 62, Copyright (2019), with permission from Elsevier. 
Whereas the above examples focused on controlled drug delivery, bio-based particle/hydrogel composites have also been employed for other biomedical applications. Tissue engineering applies the principles of biology and engineering to the development of functional substitutes for damaged tissue. ${ }^{64}$ From a biological point of view, hydrogels are attractive candidates for tissue engineering constructs because they can provide an aqueous 3D environment for cells, simulating the natural extracellular matrix. Also, viscoelastic properties of hydrogels can be tuned to influence cell fate and activity. Hybrid approaches offer unprecedented opportunities to mimic biological tissues with biomaterials. ${ }^{65,} 66$ In particular, the incorporation of nano- or microparticles in hydrogels may enhance their mechanical properties and their biological performance. To mimic the complex nature of tissues, additive manufacturing has emerged as a valuable technology to generate bioengineered $3 \mathrm{D}$ structures. ${ }^{67} \mathrm{In}$ this approach, termed biofabrication, biological structures for tissue engineering are created by a computer-aided manufacturing process for patterning and assembling living and non-living materials with a prescribed 3D organization. The inks for biofabrication are typically based on hydrogels. However, 3D printed hydrogel scaffolds often suffer from low printing accuracy and poor mechanical properties because of their soft nature and tendency to shrink. To address this challenge, Zhang et al. prepared composite scaffolds by incorporating silk microparticles in chitosan-based bioinks (Figure 3). ${ }^{68}$ A bioprinter was used to extrude the composite bioink layer-by-layer, followed by manually pipetting $\mathrm{NaOH}$ solution onto the printed filaments to solidify the construct via crosslinking of the chitosan chains. In comparison with pure chitosan scaffolds, the addition of silk microparticles resulted in a 5-fold increase of the compressive modulus as well as an improved printing accuracy and a higher resistance against degradation. To better understand the improvement in mechanical properties resulting from the presence of silk microparticles, the scaffold stiffness data was fitted to existing empirical models for the prediction of the performance of particle-polymer composites. It was found that the silk 
microparticles led to a modulus enhancement that matched those predicted for longer fibers, confirming that the particles act as very efficient reinforcing agents in chitosan scaffolds. The composite hydrogel scaffolds showed no cytotoxicity and supported adherence and growth of human fibroblast cells.
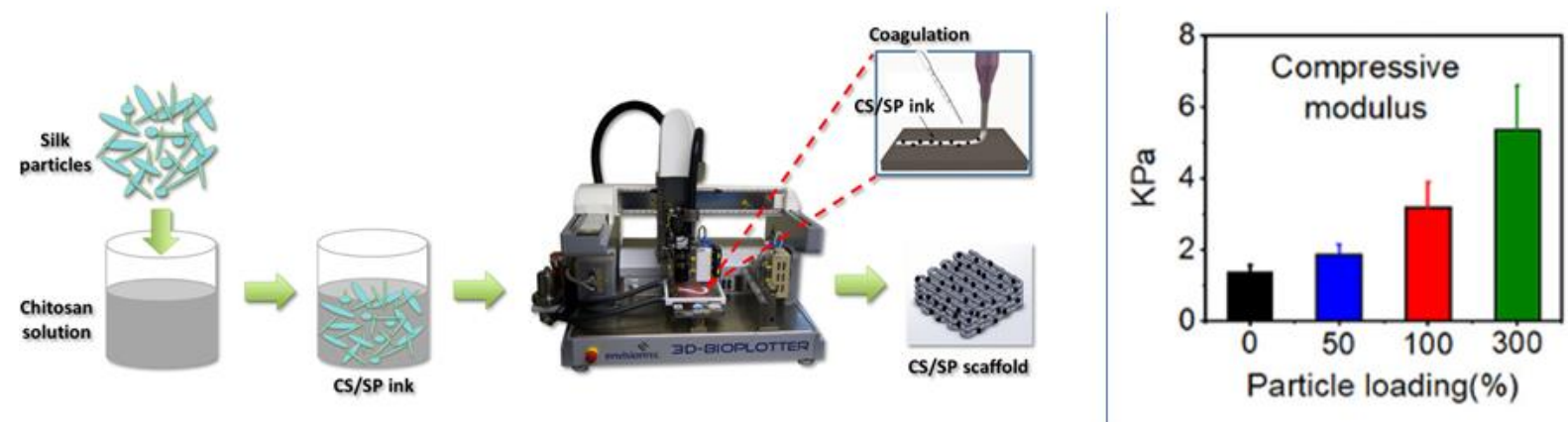

Figure 3. Left panel: Schematic representation of the silk microparticle/chitosan hydrogel printing process. Right panel: Compressive modulus of silk microparticle/chitosan hydrogel composites as a function of particle loading. Reprinted (adapted) with permission from reference 68. Copyright 2018 American Chemical Society.

Whereas the number of publications on 3D printed hydrogels for tissue engineering is rapidly increasing, research concerning drug delivery from these constructs is still very limited, especially in the case of 3D printed bio-based composite hydrogels. Poldervaart et al. developed a hydrogel scaffold for bone tissue engineering based on calcium crosslinked alginate. ${ }^{69}$ The bone growth factor BMP-2 was loaded in glutaraldehyde-crosslinked gelatin microspheres, which were in turn dispersed in the alginate bioink prior to biofabrication. Using this approach a more sustained BMP-2 release was achieved in vitro in comparison with direct incorporation of BMP-2 in the alginate hydrogel precursor. The BMP-2 retained its bioactivity after biofabrication as demonstrated by in vitro osteogenic differentiation of embedded goat multipotent stromal cells. The release of BMP-2 from the 3D printed hydrogels significantly 
stimulated bone formation in vivo, but the loading of BMP-2 in the gelatin microspheres instead of direct loading in the hydrogel did not have an influence on the bone growth in the scaffolds.

\section{$\underline{\text { 4. Bio-based composites of electrospun fibers and hydrogels }}$}

The high water content of hydrogels endows them with various advantageous properties, including biocompatibility as well as the possibility to embed and release biologically active molecules. However, using hydrogels for mechanically demanding tasks is challenging, as the large volume fraction of water may result in weakness, compliance and brittleness. In order to obtain systems with improved mechanical properties without compromising cellular viability, composites of hydrogels and reinforcing materials, most often fibers, have been developed. Electrospinning is most commonly used to produce fibrous materials because it is a straightforward and effective method to prepare thin fibers with diameters ranging from micrometres to a few nanometres at low costs and high production rates. This electrostatic processing method uses a high-voltage electric field to form solid fibers from a polymeric fluid stream delivered through a millimetre-scale nozzle. ${ }^{70}$

This section focuses on recent examples of bio-based hydrogels containing mainly noncellulose fibers; the emerging field of nanocellulose-containing bio-based hydrogels will be covered separately in the last section of this review. The reader is referred to a number of excellent reviews concerning hydrogels with incorporated electrospun fibers that focus on specific application areas such as tissue engineering, ${ }^{70-72}$ osteochondral regeneration ${ }^{73}$ and wound dressings. ${ }^{74}$

Since the mechanical performance of hydrogel scaffolds is often inferior to that of native tissues, the development of mechanically robust hydrogels is especially important to provide optimal performance in tissue engineering applications. The group of Oyen prepared fiber- 
reinforced hydrogels using gelatin and alginate to mimic the microstructure and composition of the extracellular matrix of soft tissue (Figure 4). ${ }^{75}$ Gelatin was electrospun into nanofibers and infiltrated with alginate solution, which was ionically crosslinked with calcium ions. The composites were further crosslinked using 1-ethyl-3-(-3-dimethylaminopropyl) carbodiimide / N-hydroxysuccinimide (EDC/NHS) chemistry to induce covalent bond formation. Composites contained fibers that were in a single layer or stacked in multilayer laminates with varying fiber orientations. Mechanical tests showed that the tensile strength of native alginate hydrogels (approximately $100 \mathrm{kPa}$ ) could be enhanced by up to two orders of magnitude by adding gelatin fiber mats. The larger the fraction of fibers in the direction of the applied load, the stiffer and stronger the composite hydrogels were. Without fiber reinforcement, alginate hydrogels exhibited brittle failure at small strains with a tear toughness of $40 \mathrm{~J} / \mathrm{m}^{2}$. Incorporation of gelatin fibers as multilayer laminates with different fiber orientations for each layer increased the toughness by two orders of magnitude compared with the unreinforced hydrogel. This was explained by a fiber-induced crack-deflecting mechanism which dissipates energy and locally reduces stresses. In addition, interfacial debonding was stated as energy dissipating mechanism that contributes to the increase in toughness. The composite gelatin fiber/alginate gel scaffolds closely matched the mechanical properties and transparency of porcine cornea, showing their potential for corneal tissue engineering. ${ }^{76}$ 


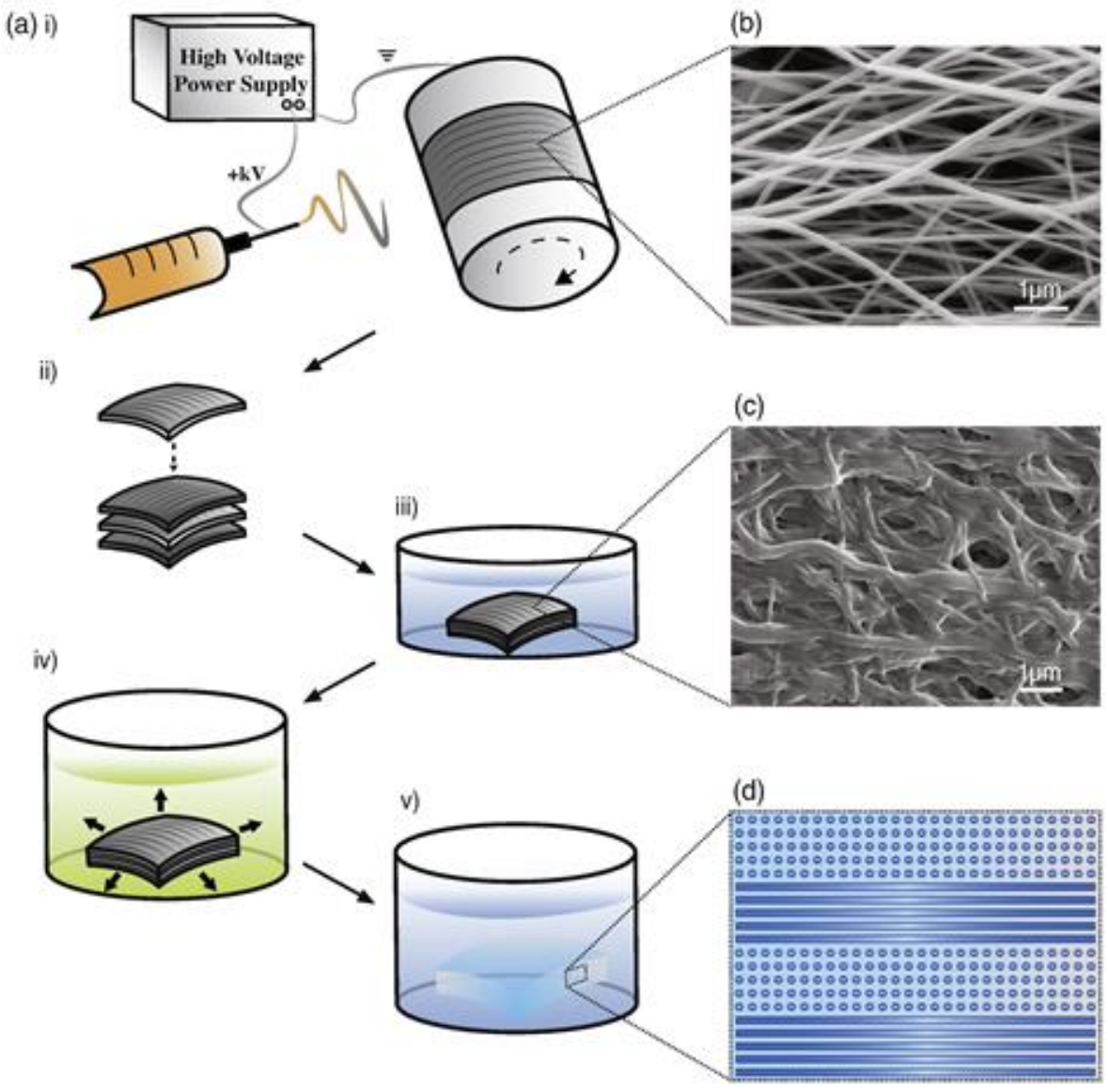

Figure 4. (a) Schematic representation of the fabrication of gelatin fiber/alginate hydrogel composites. i) electrospun fibers are collected on a rotating drum to define the dominant fiber direction, ii) stacked with varying fiber orientations, iii) crosslinked in ethanol using EDC/NHS, iv) submerged in an aqueous alginate solution, which is v) gelled in $\mathrm{CaCl}_{2}$ solution followed by additional EDC/NHS crosslinking. (b) Scanning electron microscopy (SEM) image of aligned gelatin nanofibers after collection from the rotating drum (a,i). (c) SEM image of the electrospun gelatin fibers following the first crosslinking step (a,iii). (d) Illustration of a typical multilayer laminate hydrogel composite $(\mathrm{a}, \mathrm{v})$ with 4 orthogonal layers of aligned gelatin in a cross-ply $0^{\circ} / 90^{\circ} / 0^{\circ} / 90^{\circ}$ fiber orientation pattern. Reproduced with permission from reference 75. 
Other recent examples of fiber-reinforced bio-based composites for tissue engineering include PLA fiber/chitosan hydrogel, ${ }^{77}$ PLA fiber/hyaluronic acid-graft-alginate hydrogel ${ }^{78}$ and collagen fiber/alginate hydrogel ${ }^{79}$ systems.

The preparation of mechanically robust, injectable hydrogels can be challenging because the hydrogel precursors may diffuse away from the site of injection, thereby decreasing the effective polymer concentration. In addition, the in vivo setting limits the application of highly reactive, toxic crosslinking agents and end groups. With the goal of enhancing the mechanical properties of injectable gelatin hydrogels, Poveda-Reyes and co-workers investigated two methods for the fabrication of reinforcing fibers made from PLA (Figure 5). ${ }^{80}$ One method consisted of manufacturing electrospun meshes followed by milling, whereas the second method involved precipitating a PLA solution in a highly turbulent non-solvent bath. In both cases, fibers of approximately $50 \mu \mathrm{m}$ in length and $1 \mu \mathrm{m}$ in diameter were obtained. To prepare composites, the fibers were dispersed in a solution of tyramine-grafted gelatin which was subsequently enzymatically crosslinked using hydrogen peroxide and horseradish peroxidase. At low concentrations $(<1.5 \mathrm{w} / \mathrm{v} \%)$ no limitations regarding injectability were observed and the high crosslinking rate (gelation time $<1 \mathrm{~min}$ ) prevented the precipitation of the fibers in the hydrogel precursor solution. A triple increase in the Young's modulus of the gelatin hydrogel was obtained when electrospun, milled fibers were incorporated, which was explained by a reduction in the water permeability and flow in the hydrogels. In contrast, no enhancement in Young's modulus was observed when precipitation-fabricated fibers were embedded. This was ascribed to the heterogeneous morphology and rough surface of the fibers leading to the formation of fiber aggregates, which do not influence water flow in the hydrogel. Cells were incorporated in the composites by adding them to the gelatin-fiber dispersions prior to 
crosslinking. It was found that the injectable systems hydrogels are not cytotoxic and induce steady cell growth.
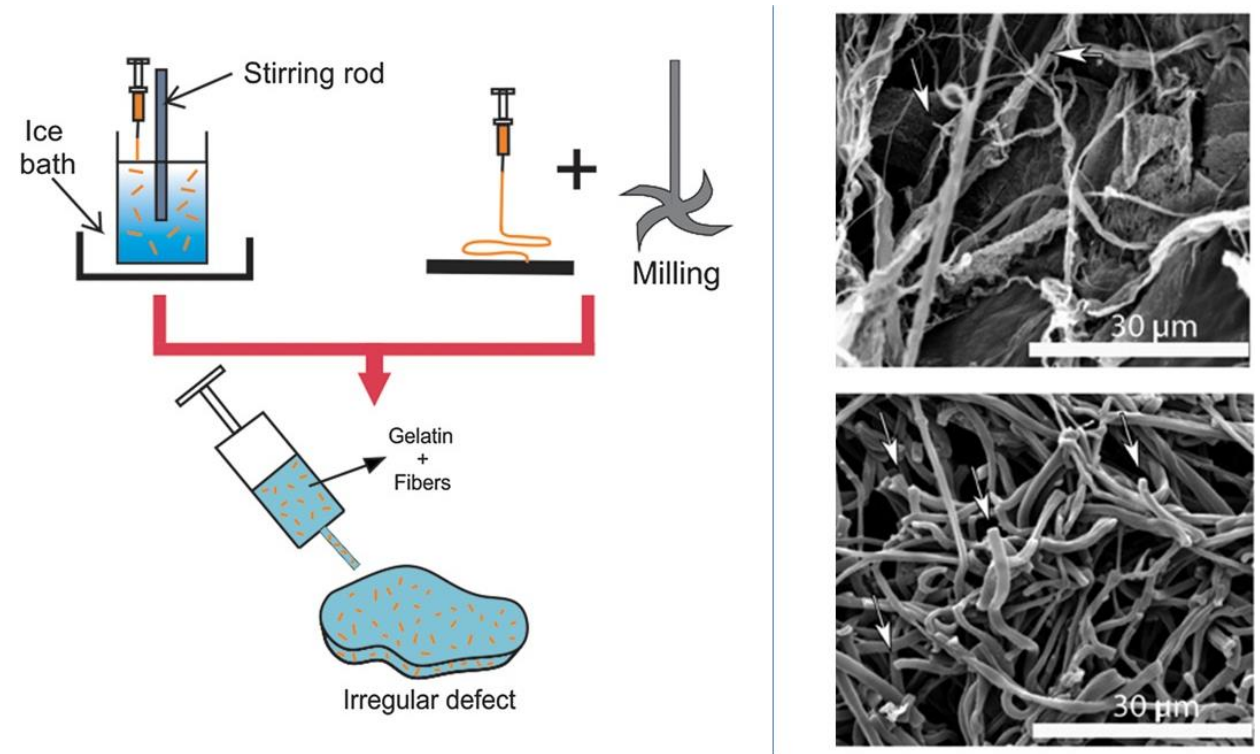

Figure 5. Left panel: Schematic illustration of the preparation of injectable PLA fiber/gelatin hydrogel composites with two distinct routes for the fabrication of the PLA fibers. Right panel: SEM images of PLA fibers prepared via precipitation (top) and electrospun, milled PLA fibers (bottom). Adapted from reference 80, (C) 2015 WILEY-VCH Verlag GmbH \& Co. KGaA, Weinheim.

Other in situ forming bio-based composite hydrogels, including silk fiber/chitosan hydrogel ${ }^{81}$ and albumin fiber/gelatin hydrogel ${ }^{82}$ systems, similarly demonstrated suitable mechanical properties for tissue engineering while maintaining biocompatibility.

In addition to enhancing the mechanical properties of native, single component hydrogels, the incorporation of electrospun fiber mats in hydrogels can also be exploited to control the behaviour of incorporated cells. This was demonstrated by Yang et al., who embedded highly aligned electrospun PLA nanofiber meshes in collagen hydrogels via a bottom-up, layer-by- 
layer assembly process, resulting in highly organized composite hydrogels (Figure 6). ${ }^{83}$ The fibers dictated the orientation of the cytoskeleton of individual cells in a precise manner, allowing for control over the orientation of a cell population throughout the thickness of the hydrogel. The addition of fibers could also be used to direct the cell phenotype and the secretion of matrix proteins.
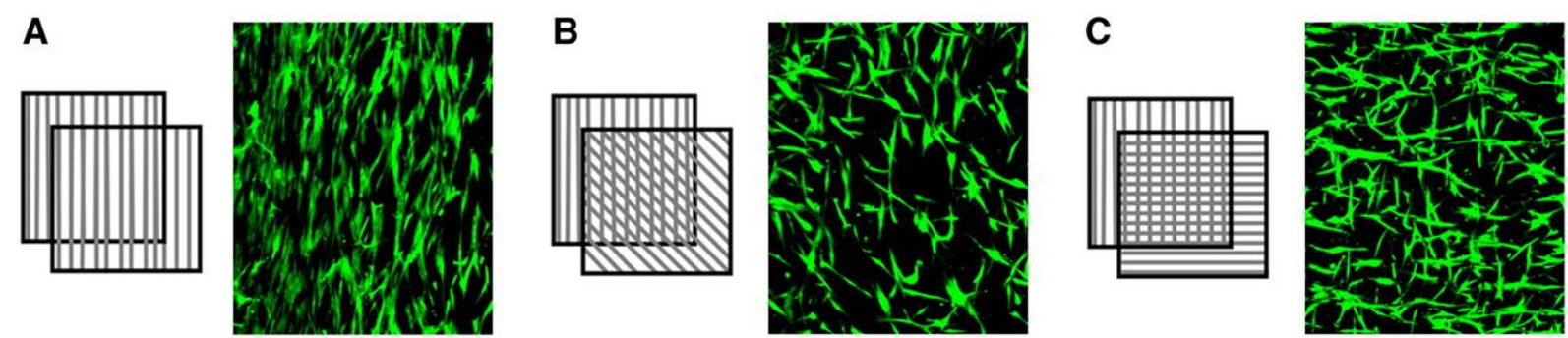

Figure 6. Confocal laser scanning microscopy images of fibroblasts grown in PLA fiber/collagen hydrogel composites with the fiber meshes oriented (A) in parallel, (B) at $45^{\circ}$ and (C) perpendicular to each other. Reprinted from reference 83, Copyright (2011), with permission from Elsevier.

Although bio-based polymers generally show good biocompatibility when they are applied as macroscopic biomaterials, the nanotopological features of bio-based polymeric fibers, including diameter and surface morphology, may negatively influence cell behaviour. This was demonstrated by Shoichet and co-workers, who reported that the presence of electrospun collagen fibers in hyaluronic acid/methylcellulose hydrogels was deleterious to neural stem/progenitor cell (NSPC) proliferation and differentiation. ${ }^{84}$ In contrast, composites with synthetic poly( $\varepsilon$-caprolactone-co-lactide) fibers maintained NSPC viability and guided cellular differentiation to neurons and oligodendrocytes, similar to native hydrogels. The inability of incorporated collagen fibers to support differentiation of NSPCs was attributed to their fine, fragmented and tangled structures. This demonstrates that the nanotopological characteristics 
of fibers should be taken into account as an important parameter when designing and evaluating bio-based fiber/hydrogel composites for tissue engineering applications.

Bioadhesives and sealants for wound closure and healing applications are promising alternatives when other techniques, such as stapling or suturing, are impractical or inefficient. Pinkas et al. investigated the effect of cellulose fibers on the properties of a hydrogel formulation based on a combination of gelatin and alginate which was crosslinked with a watersoluble carbodiimide. ${ }^{85}$ The incorporation of fibers (average length of $300 \mu \mathrm{m}$ ) significantly increased the cohesive properties of the hydrogel, most notably the burst strength i.e. the maximum pressure the hydrogel could withstand. This was ascribed to penetration of the gelatin and alginate formulation into pores of the cellulose fibers, enabling a good fiber-matrix interaction. Although the adhesive properties of the composite hydrogel were not tested under biologically relevant conditions, in previous studies the non-reinforced gelatin-alginate hydrogel showed good bonding strength to porcine skin as well as a high biocompatibility, ${ }^{86,87}$ suggesting the potential of these fiber-reinforced composite hydrogels for bioadhesive and sealant applications.

In the field of controlled drug release, obtaining extended, uniform release with little initial burst has been challenging, especially for macromolecular therapeutics such as antibodies. To address this challenge, the preparation of electrospun fiber/hydrogel composite materials is an attractive approach, since the electrospun fiber mat may provide an additional diffusion barrier that can alter the release kinetics. ${ }^{88}$ Elia et al. prepared composites of electrospun silk fibroin mats and hydrogels formed by crosslinking thiol-modified hyaluronic acid with poly(ethylene glycol)-diacrylate. ${ }^{89}$ Release experiments were performed using fluorescein isothiocyanatedextran (FITC-dextran) as a model compound in the presence of the enzyme hyaluronidase, 
which is capable of degrading hyaluronic acid. Low molecular weight FITC-dextran diffused freely and quickly out of the highly porous hydrated gel, whereas high molecular weight FITCdextran was released more slowly and involved both diffusion and enzymatic degradation of the hydrogel. To further evaluate the feasibility of the composites for controlled drug delivery, several anti-inflammatory drugs such as dexamethasone and prednisolone were dissolved in the precursor solutions prior to crosslinking and their in vitro release was measured in the absence of enzymes. All drugs exhibited first order release kinetics and complete release was achieved in several hours or several days, depending on the drug size and hydrophilicity. The observed release rates from the composite hydrogels were lower than those from hydrogels lacking a fiber mat, indicating that the drugs were retained for a longer period of time in the composite systems. This was ascribed to direct drug entrapment within the silk fibers or to swelling of the fibers, thereby hindering the release of drug near the silk by acting as a diffusion barrier. In addition to the relatively fast release of anti-inflammatory drugs, the composites provided a sustained release of vascular endothelial growth factor, a signal protein, for several weeks.

\section{Bio-based composites of nanocellulose and hydrogels}

Natural plant and wood fibers can be processed via mechanical, chemical and enzymatic treatments to yield micro- and nanoscopic fibrils with altered mechanical properties. If the diameter of the fibrils is decreased to the nanoscale, they may exhibit remarkable reinforcing effects thanks to the presence of crystalline regions with a very high mechanical strength. Generally two types of such cellulose-based nanostructures are distinguished: cellulose nanocrystals and cellulose nanofibrils (Figure 7). Cellulose nanocrystals are elongated, rigid rod-shaped particles with a diameter of 2-20 nm and a length of 100-600 nm. ${ }^{90}$ They have been described in literature with various terms, including nanowhiskers and nanocrystalline cellulose. Cellulose nanofibrils, on the other hand, are more flexible and longer (diameter of 
10-40 nm, length $>1 \mu \mathrm{m})$. Cellulose nanofibrils have also been referred to in literature as nanofibrillated cellulose, microfibrillated cellulose or microfibrils, despite their nanoscale dimensions.
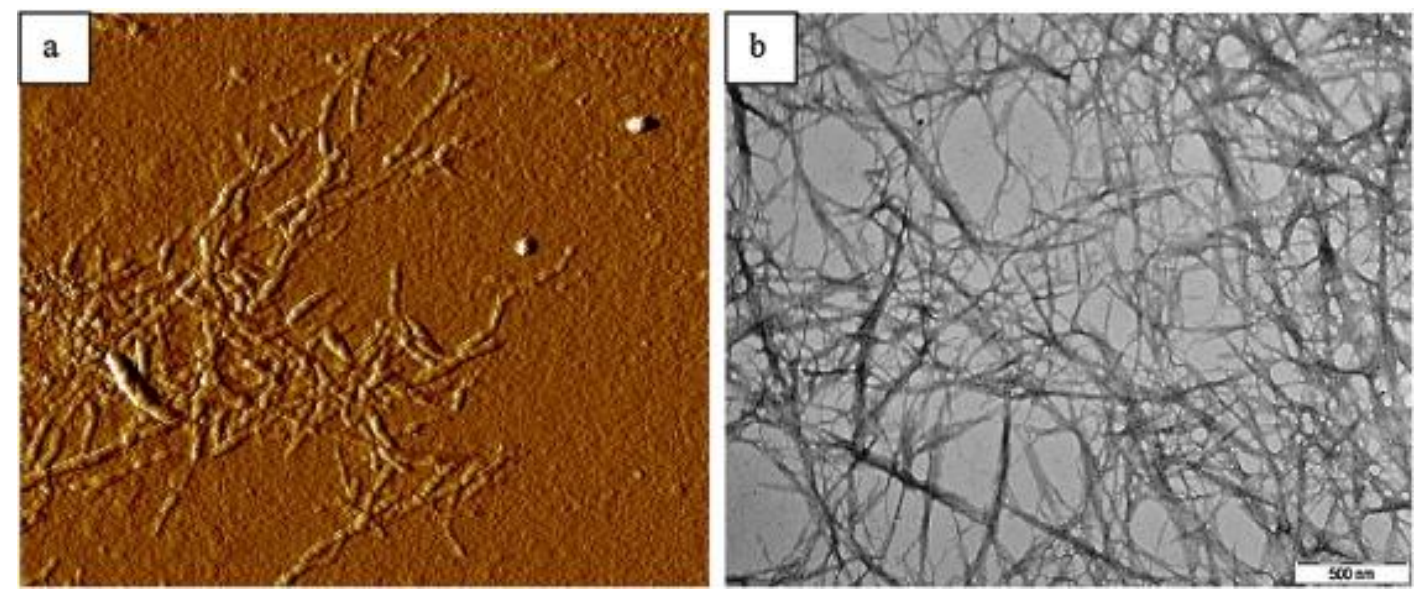

Figure 7. Examples of cellulose nanostructures obtained from the bast of the kenaf plant: (a) Atomic force microscopy image of cellulose nanocrystals and (b) transmission electron microscopy image of cellulose nanofibrils. Reprinted from reference 91, Copyright (2016), with permission from Elsevier.

Because of their high mechanical strength, biocompatibility and biodegradability, both cellulose nanocrystals and cellulose nanofibrils have been incorporated in hydrogels to efficiently impart mechanical reinforcement as well as various other desirable properties. Nanocellulose concentrations as low as $5 \mathrm{wt} \%$ have been shown to significantly enhance the mechanical properties of calcium crosslinked alginate hydrogels, whereas higher loadings did not lead to further improvements. ${ }^{92}$ In addition to vegetal sources, nanocellulose can also be obtained from certain gram-negative bacteria such as Gluconacetobacter xylinus. Bacterial cellulose consists of a 3D network of interconnected cellulose nanofibers and is characterized by high strength and purity. ${ }^{93}$ 
The field of nanocellulose-containing hydrogels has been reviewed a number of years ago by De France et al. ${ }^{94}$ The following section focuses on selected, recent examples of the use of nanocellulose in bio-based composite hydrogels for biomedical applications.

Thanks to their reinforcing effects, cellulose nanostructures have been frequently embedded in hydrogels to create substitutes for intervertebral discs. Pereira et al. prepared composite hydrogels by dispersing cellulose nanocrystals (100 nm in length) in gellan-gum precursor solutions. ${ }^{95}$ The addition of phosphate buffered saline induced gelation by reduction of gellan gum helix repulsion via coordination of sodium and potassium ions with carboxylate groups on the helices. The critical gel concentration of gellan-gum decreased in the presence of cellulose nanocrystals. This was explained by the entanglement of cellulose nanocrystals between gellangum helices resulting in molecular bridging and chain crosslinking. The presence of cellulose nanocrystals enhanced the mechanical properties of the hydrogels such as the storage modulus and ultimate stress under compression. Furthermore, the composites matched the Young's modulus $(50 \mathrm{kPa})$ of native human intervertebral disc tissue. All hydrogels exhibited a slow degradation rate (5-10\% mass loss after 30 days), which was sufficient to allow free motion of bovine annulus fibrosus cells in the constructs. Cells cultured in the composite hydrogels showed a physiologically more relevant cell morphology (elongation and spreading) compared to cells cultured in the native gellan-gum hydrogels, which kept a round morphology after 14 days.

Similar promising results for intervertebral disc regeneration were obtained by Doench and coworkers for physically crosslinked chitosan hydrogels reinforced with cellulose nanofibrils, ${ }^{96}$ thereby exploiting the excellent compatibility of chitosan with cellulose. ${ }^{91,97}$ Notably, ex vivo experiments performed in a pig vertebral unit model showed that the implantation of the 
composite hydrogel in a disc defect contributes to the restoration of the disc biomechanics, thereby approaching the functionality of a healthy disc.

In an attempt to improve the mechanical properties of nanocellulose-containing chitosan hydrogels for intervertebral disc regeneration and to better control their degradation, the group of Ulery applied a dual crosslinking technique by combining ionic crosslinking (using calcium phosphate) with covalent crosslinking via Schiff base formation (using genipin), resulting in a semi-interpenetrating network structure of nanocellulose and dual crosslinked chitosan. ${ }^{98}$ As expected, ionic crosslinking, covalent crosslinking and the incorporation of cellulose nanocrystals all had an incremental effect on the compressive strength, reaching values comparative to human vertebral bone for some formulations (250-300 kPa). Composites with a very high crosslink density showed an even higher compressive strength but also brittleness and a fast degradation, making them less suitable for intervertebral disc tissue regeneration. Although this system allows for a high control over the composite hydrogel properties thanks to the various reinforcement strategies (dual crosslinking as well as the incorporation of nanocellulose), the observed cytotoxicity of the hydrogel, which may be related to the use of genipin as a covalent crosslinker, needs to be addressed for clinical translation.

A different example of a robust semi-interpenetrating network was reported by Hossen et al., ${ }^{99}$ who incorporated cellulose nanofibrils in a solution of methacrylate-functionalized carboxymethyl cellulose (Figure 8). Subsequent UV irradiation led to photopolymerization of the methacrylate groups and, after freeze-drying, highly porous structures. The observed high resistance against degradation and collapse of the porous structure was ascribed to the presence of cellulose nanofibrils and chemical crosslinks, but also to hydrogen bonding between the two cellulose species present in these composite hydrogels. 
a

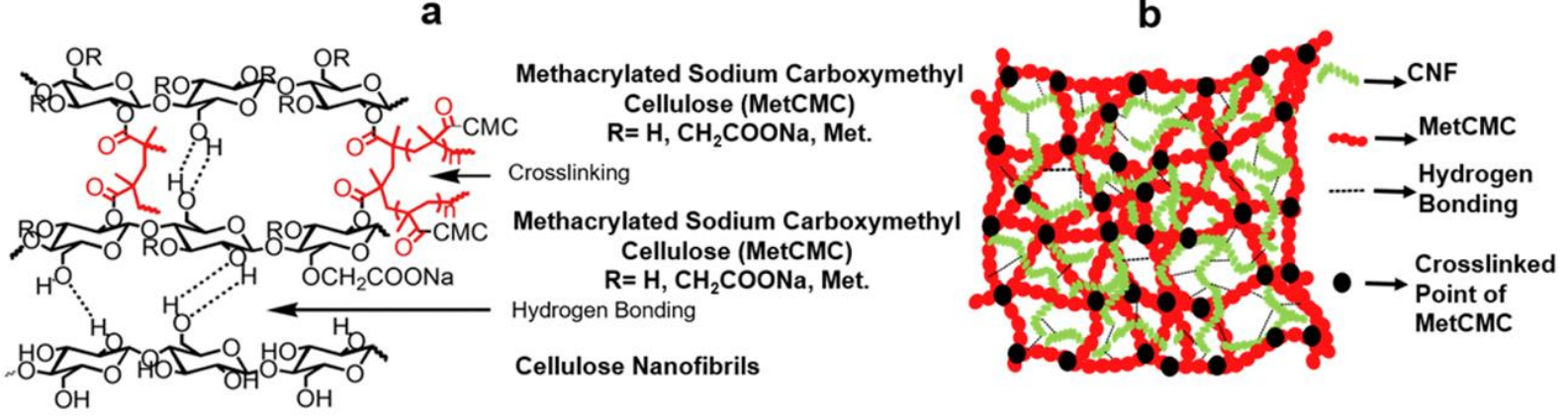

Figure 8. Schematic illustration of the crosslinking mechanisms in composites consisting of cellulose nanofibrils embedded in methacrylated carboxymethyl cellulose hydrogels. Reprinted from reference 99, Copyright (2018), with permission from Elsevier.

Nanocellulose has also gained interest for 3D printing of hydrogels because of its ability to improve shape reliability while printing. Most research to date focused on composites of cellulose nanofibrils or cellulose nanocrystals in alginate-based bioinks. Sultan et al. reported on a composite bioink consisting of cellulose nanocrystals in an alginate/gelatin hydrogel (Figure 9). ${ }^{100}$ The composite bioink exhibited shear thinning behavior, as the viscosity decreased by four orders of a magnitude after increasing the shear rate to values typically encountered during 3D printing. The native alginate/gelatin formulation showed no shear thinning behaviour, suggesting that this formulation itself is not printable. The $3 \mathrm{D}$ printed structures were immersed in calcium chloride solution to ionically crosslink the alginate and subsequently in a glutaraldehyde solution to covalently crosslink the gelatin. SEM and X-ray scattering studies showed that the cellulose nanocrystals align preferably in the printing direction, which may be useful for guided cell growth during tissue regeneration. 


\section{i) Hydrogel ink preparation}

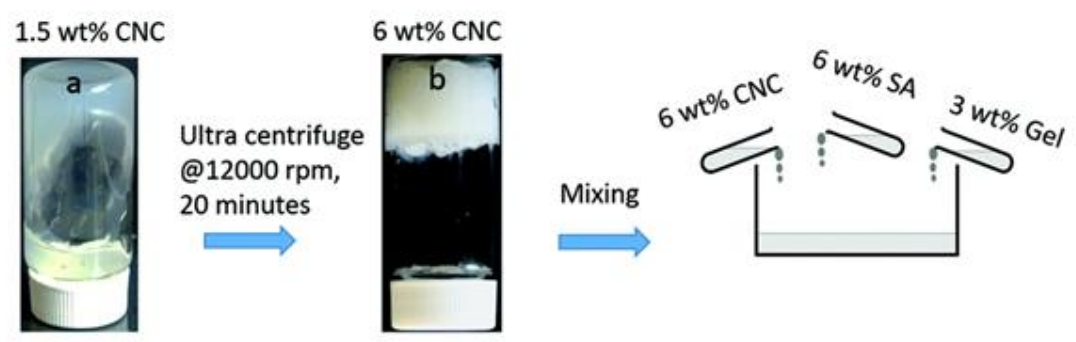

\section{ii) 3D Printing}

Nanocomposite hydrogel ink

3D printed hydrogel scaffolds
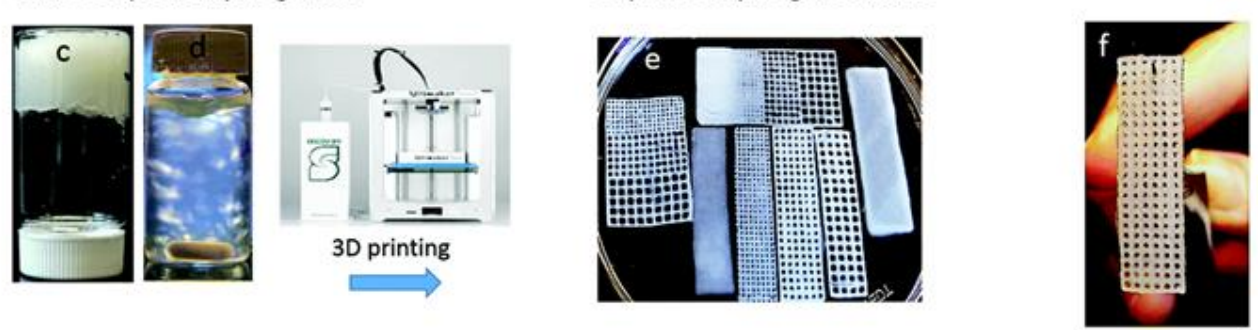

\section{iii) Crosslinking}

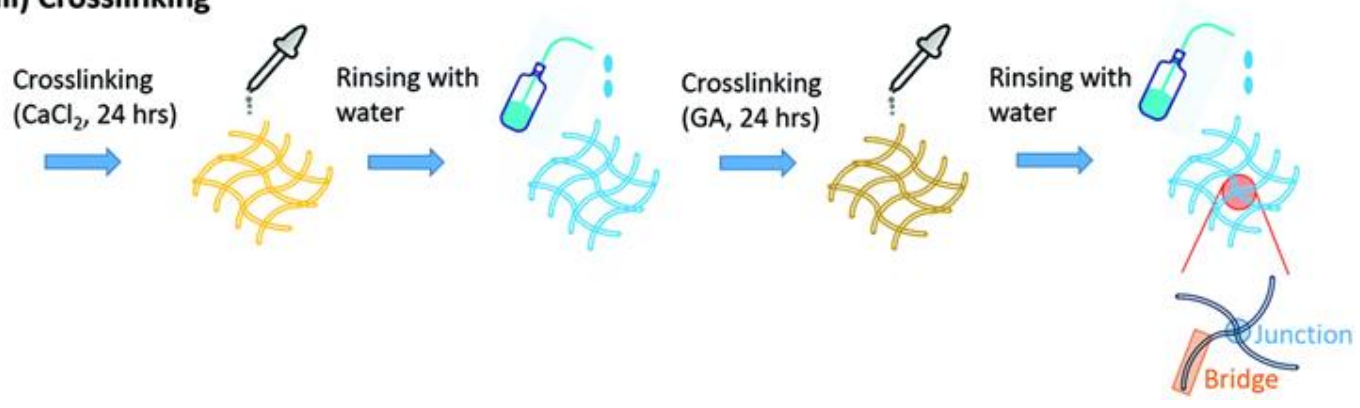

Figure 9. 3D printing of composite scaffolds consisting of cellulose nanocrystals in alginate/gelatin hydrogels. (i) Preparation of the composite bioink with (a) cellulose nanocrystal gel at $1.5 \mathrm{w} / \mathrm{v} \%$ and (b) at $6 \mathrm{w} / \mathrm{v} \%$. (ii) 3D printing process, showing (c) a photograph of the composite bioink, (d) the flow birefringence of the bioink at $10 \mathrm{x}$ dilution, (e) 3D printed porous composite hydrogels and (f) the stability of 3D printed porous composite hydrogels after crosslinking. (iii) Crosslinking methods for post-printing stabilization of the 3D printed composite hydrogels. Reproduced with permission from reference 100. Published by The Royal Society of Chemistry.

Other studies have demonstrated the potential of nanocellulose/alginate composite bioinks for 3D bioprinting of bone ${ }^{101}$ and cartilage tissue. ${ }^{102,103}$ When pluripotent stem cells and human 
chondrocytes were incorporated in such bioinks during the 3D printing process, cartilaginous tissue as well as largely increased cell numbers were observed in the composite scaffolds after 5 weeks. ${ }^{104}$

Nanocellulose-containing bio-based hydrogels have been frequently investigated for tissue engineering, as exemplified above; comparatively much less publications deal with controlled drug delivery from such composites. $\mathrm{Xu}$ et al. reported on the fabrication of cellulose nanocrystal/chitosan hydrogels for the controlled delivery of the hydrophilic respiratory drug theophylline. ${ }^{105}$ Cellulose nanocrystals were first oxidized using sodium periodate to obtain dialdehyde nanocellulose. Then, chitosan was crosslinked via Schiff base formation using dialdehyde nanocellulose in different weight ratios to fabricate cellulose nanocrystal/chitosan hydrogel composites. When the chitosan percentage in the hydrogel was increased, the isoelectric point shifted towards a higher $\mathrm{pH}$, likely as a result of a higher amount of free amino groups. The lowest degree of swelling for a given formulation occurred at the isoelectric point, where the electrostatic repulsion between the ionic charges in the network is minimal. The degree of swelling increased with decreasing nanocellulose concentration due to a lower crosslink density. The theophylline release profiles showed a burst release during the first few hours, after which the drug release levelled off. The release rate decreased as the dialdehyde nanocellulose content in the composites increased, resulting from a higher amount of crosslinks in the networks. Due to a higher swelling ratio of the hydrogels in an acid environment, the cumulative drug release at $\mathrm{pH} 1.5(85 \%)$ was significantly higher than the cumulative drug release at $\mathrm{pH} 7.4(25 \%)$, showing the potential of these composite hydrogels as gastric drug delivery systems.

Following a similar approach, Zheng et al. prepared giant network composite hydrogels by reacting dialdehyde nanocellulose with gelatin via Schiff base formation. ${ }^{106}$ Upon incorporation 
of the nanocellulose, the compression strength of the hydrogel increased to $1.6 \mathrm{MPa}, 40$ times that of the pure gelatin hydrogel.

Only a limited number of publications report on bio-based hydrogel composites containing cellulose nanofibers obtained from bacteria. For example, Park et al. incorporated bacterial nanocellulose in alginate hydrogels and showed that these composites are potential candidates for cell encapsulation thanks to the 3D fibrous structure of the cellulose, which resembles the extracellular matrix. ${ }^{107}$ Yan et al. demonstrated that the addition of bacterial nanocellulose to alginate/collagen hydrogels resulted in improved compressive strength as well as reduced hydrogel swelling and degradation. ${ }^{108}$ This was ascribed to the fibrillar meshwork structure of bacterial nanocellulose and the formation of intermolecular hydrogen bonding among the components of the composite hydrogel. Interestingly, cellulose-producing bacteria offer the possibility for in situ biosynthesis of nanocellulose/hydrogel composites by supplementing the bacterial culture medium with hydrogel precursors. ${ }^{109,110}$

\section{Conclusions and outlook}

Important progress has been made in the field of bio-based hydrogels for biomedical applications during the last decades. This review clearly shows the interest of incorporating an additional component in such hydrogels, as the resulting composite materials present markedly improved, synergistic properties. In particular, incorporation of micelles or microspheres in hydrogels facilitates the simultaneous, controlled release of multiple drugs with different solubilities, including hydrophobic drugs. Furthermore, the inclusion of electrospun fibers in hydrogels enhances their mechanical properties, enabling their use as robust tissue engineering scaffolds. Nanocellulose, an emerging class of materials encompassing both nanowhiskers and nanofibrils, has shown particularly effective in reinforcing hydrogels at remarkably low 
concentrations due to the presence of crystalline cellulose regions with a very high mechanical strength. In addition to facilitating desired drug release profiles and structural properties, particles and fibers have also been used to improve the biological performance of composite hydrogels by optimizing the micro-environmental conditions for cells.

Despite the vast progress that has been achieved in the field, a number of challenges remain that should be overcome in order to translate bio-based composite hydrogels into viable biomedical solutions. First of all, most of the bio-based composite hydrogels have only been evaluated in vitro. To enable clinical translation of these promising systems, a strong need exists to study the therapeutic effects in vivo together with their long-term biodegradability, toxicity and accumulation. In addition, many of the composite hydrogels discussed in this review concern systems that have to be formed into their final shape before implantation. However, in situ hydrogel formation can be regarded as an important requirement in view of the clear advantages such as facile administration, improved patient comfort and the straightforward incorporation of therapeutic agents as well as particles or fibers. In this respect, a significant and fast response to environmental gelation triggers such as temperature (for physical crosslinking) or the use of bioorthogonal reactive groups, which do not interfere with biological processes upon covalent crosslinking, are considered crucial. Lastly, although the field of biobased composite hydrogels is rapidly evolving, as demonstrated in this review, the number of reports on systems displaying spatial and temporal control over drug release and/or cell behavior is still very limited. In this regard, 3D printing is a valuable tool since it enables the fabrication of gradient composites with a defined architecture and regional differences, which potentially allows for a high level of control over drug release and cellular responses.

In summary, thanks to their inherent biocompatibility, their versatile nature as well as the increasing demand for products from renewable resources, bio-based composite hydrogels will 
likely increasingly replace single component, synthetic hydrogels in biomedical applications in the years to come.

\section{Acknowledgements}

The author gratefully acknowledges Dr. Tatiana Budtova for critical reading and her suggestions for improvement of the manuscript.

\section{References}

[1] S.J. Buwalda, T. Vermonden, W.E. Hennink, Hydrogels for therapeutic delivery: current developments and future directions, Biomacromolecules 18 (2017) 316-330.

[2] S.J. Buwalda, K.W.M. Boere, P.J. Dijkstra, J. Feijen, T. Vermonden, W.E. Hennink, Hydrogels in a historical perspective: from simple networks to smart materials, Journal of Controlled Release 190 (2014) 254-273.

[3] T. Vermonden, B. Klumperman, The past, present and future of hydrogels, European Polymer Journal 72 (2015) 341-343.

[4] J. Kopecek, Hydrogels: From soft contact lenses and implants to self-assembled nanomaterials, Journal of Polymer Science Part A: Polymer Chemistry 47 (2009) 5929-5946.

[5] A.S. Hoffman, Hydrogels for biomedical applications, Advanced Drug Delivery Reviews 54 (2002) 3-12.

[6] H. Nakajima, P. Dijkstra, K. Loos, The recent developments in biobased polymers toward general and engineering applications: polymers that are upgraded from biodegradable polymers, analogous to petroleum-derived polymers, and newly developed, Polymers 9 (2017) 523.

[7] R.P. Babu, K. O'connor, R. Seeram, Current progress on bio-based polymers and their future trends, Progress in Biomaterials 2 (2013) 8. 
[8] S. Van Vlierberghe, P. Dubruel, E. Schacht, Biopolymer-based hydrogels as scaffolds for tissue engineering applications: a review, Biomacromolecules 12 (2011) 1387-1408.

[9] A. Salerno, C.D. Pascual, Bio-based polymers, supercritical fluids and tissue engineering, Process Biochemistry 50 (2015) 826-838.

[10] F. Zhao, D. Yao, R. Guo, L. Deng, A. Dong, J. Zhang, Composites of polymer hydrogels and nanoparticulate systems for biomedical and pharmaceutical applications, Nanomaterials 5 (2015) 2054-2130.

[11] A. Lendlein, R.S. Trask, Multifunctional materials: concepts, function-structure relationships, knowledge-based design, translational materials research, Multifunctional Materials 1 (2018) 010201.

[12] K.Y. Lee, D.J. Mooney, Alginate: properties and biomedical applications, Progress in Polymer Science 37 (2012) 106-126.

[13] T. Coviello, P. Matricardi, F. Alhaique, Drug delivery strategies using polysaccharidic gels, Expert Opinion on Drug Delivery 3 (2006) 395-404.

[14] A.D. Augst, H.J. Kong, D.J. Mooney, Alginate hydrogels as biomaterials, Macromolecular Bioscience 6 (2006) 623-633.

[15] T. Vermonden, R. Censi, W.E. Hennink, Hydrogels for protein delivery, Chemical Reviews 112 (2012) 2853-2888.

[16] A. Sannino, C. Demitri, M. Madaghiele, Biodegradable cellulose-based hydrogels: design and applications, Materials 2 (2009) 353-373.

[17] C. Chang, L. Zhang, Cellulose-based hydrogels: present status and application prospects, Carbohydrate Polymers 84 (2011) 40-53.

[18] D. Ciolacu, C. Rudaz, M. Vasilescu, T. Budtova, Physically and chemically cross-linked cellulose cryogels: structure, properties and application for controlled release, Carbohydrate Polymers 151 (2016) 392-400. 
[19] X. Shen, J.L. Shamshina, P. Berton, G. Gurau, R.D. Rogers, Hydrogels based on cellulose and chitin: fabrication, properties, and applications, Green Chemistry 18 (2016) 53-75.

[20] S.F. Kabir, P.P. Sikdar, B. Haque, M.R. Bhuiyan, A. Ali, M. Islam, Cellulose-based hydrogel materials: chemistry, properties and their prospective applications, Progress in Biomaterials 7 (2018) 153-174.

[21] V.K. Thakur, M.K. Thakur, Processing and characterization of natural cellulose fibers/thermoset polymer composites, Carbohydrate Polymers 109 (2014) 102-117.

[22] O. Korhonen, D. Sawada, T. Budtova, All-cellulose composites via short-fiber dispersion approach using $\mathrm{NaOH}-$ water solvent, Cellulose 26 (2019) 4881-4893.

[23] M. Yen, J. Yang, J. Mau, Physicochemical characterization of chitin and chitosan from crab shells, Carbohydrate Polymers 75 (2009) 15-21.

[24] A. Chenite, M. Buschmann, D. Wang, C. Chaput, N. Kandani, Rheological characterisation of thermogelling chitosan/glycerol-phosphate solutions, Carbohydrate Polymers 46 (2001) 3947.

[25] L. Cao, B. Cao, C. Lu, G. Wang, L. Yu, J. Ding, An injectable hydrogel formed by in situ cross-linking of glycol chitosan and multi-benzaldehyde functionalized PEG analogues for cartilage tissue engineering, Journal of Materials Chemistry B 3 (2015) 1268-1280.

[26] M.J. Moura, H. Faneca, M.P. Lima, M.H. Gil, M.M. Figueiredo, In situ forming chitosan hydrogels prepared via ionic/covalent co-cross-linking, Biomacromolecules 12 (2011) 32753284.

[27] Z. Shariatinia, Carboxymethyl chitosan: properties and biomedical applications, International Journal of Biological Macromolecules 120 (2018) 1406-1419.

[28] N. Bhattarai, J. Gunn, M. Zhang, Chitosan-based hydrogels for controlled, localized drug delivery, Advanced Drug delivery Reviews 62 (2010) 83-99. 
[29] J. Berger, M. Reist, J.M. Mayer, O. Felt, N. Peppas, R. Gurny, Structure and interactions in covalently and ionically crosslinked chitosan hydrogels for biomedical applications, European Journal of Pharmaceutics and Biopharmaceutics 57 (2004) 19-34.

[30] J.T. Oliveira, L. Martins, R. Picciochi, P. Malafaya, R. Sousa, N. Neves, J. Mano, R. Reis, Gellan gum: a new biomaterial for cartilage tissue engineering applications, Journal of Biomedical Materials Research Part A 93 (2010) 852-863.

[31] H. Du, P. Hamilton, M. Reilly, N. Ravi, Injectable in situ physically and chemically crosslinkable gellan hydrogel, Macromolecular Bioscience 12 (2012) 952-961.

[32] Y. Gong, C. Wang, R.C. Lai, K. Su, F. Zhang, D. Wang, An improved injectable polysaccharide hydrogel: modified gellan gum for long-term cartilage regeneration in vitro, Journal of Materials Chemistry 19 (2009) 1968-1977.

[33] X. Xu, A.K. Jha, D.A. Harrington, M.C. Farach-Carson, X. Jia, Hyaluronic acid-based hydrogels: from a natural polysaccharide to complex networks, Soft Matter 8 (2012) 32803294.

[34] M.N. Collins, C. Birkinshaw, Hyaluronic acid based scaffolds for tissue engineering - a review, Carbohydrate Polymers 92 (2013) 1262-1279.

[35] J. Lam, N.F. Truong, T. Segura, Design of cell-matrix interactions in hyaluronic acid hydrogel scaffolds, Acta Biomaterialia 10 (2014) 1571-1580.

[36] D.B. Khadka, D.T. Haynie, Protein- and peptide-based electrospun nanofibers in medical biomaterials, Nanomedicine: Nanotechnology, Biology and Medicine 8 (2012) 1242-1262.

[37] A.M. Jonker, D.W. Löwik, J.C. van Hest, Peptide-and protein-based hydrogels, Chemistry of Materials 24 (2012) 759-773.

[38] E.E. Antoine, P.P. Vlachos, M.N. Rylander, Review of collagen I hydrogels for bioengineered tissue microenvironments: characterization of mechanics, structure, and transport, Tissue Engineering Part B: Reviews 20 (2014) 683-696. 
[39] K. Su, C. Wang, Recent advances in the use of gelatin in biomedical research, Biotechnology Letters 37 (2015) 2139-2145.

[40] P. Jaipan, A. Nguyen, R.J. Narayan, Gelatin-based hydrogels for biomedical applications, MRS Communications 7 (2017) 416-426.

[41] V. Rattanaruengsrikul, N. Pimpha, P. Supaphol, In vitro efficacy and toxicology evaluation of silver nanoparticle-loaded gelatin hydrogel pads as antibacterial wound dressings, Journal of Applied Polymer Science 124 (2012) 1668-1682.

[42] B.D. Walters, J.P. Stegemann, Strategies for directing the structure and function of threedimensional collagen biomaterials across length scales, Acta Biomaterialia 10 (2014) 14881501.

[43] G.H. Altman, F. Diaz, C. Jakuba, T. Calabro, R.L. Horan, J. Chen, H. Lu, J. Richmond, D.L. Kaplan, Silk-based biomaterials, Biomaterials 24 (2003) 401-416.

[44] Z. Shao, F. Vollrath, Materials: surprising strength of silkworm silk, Nature 418 (2002) 741.

[45] A.S. Lammel, X. Hu, S. Park, D.L. Kaplan, T.R. Scheibel, Controlling silk fibroin particle features for drug delivery, Biomaterials 31 (2010) 4583-4591.

[46] J. Chutipakdeevong, U.R. Ruktanonchai, P. Supaphol, Process optimization of electrospun silk fibroin fiber mat for accelerated wound healing, Journal of Applied Polymer Science 130 (2013) 3634-3644.

[47] S. Kapoor, S.C. Kundu, Silk protein-based hydrogels: promising advanced materials for biomedical applications, Acta Biomaterialia 31 (2016) 17-32.

[48] M. McKenzie, D. Betts, A. Suh, K. Bui, L.D. Kim, H. Cho, Hydrogel-based drug delivery systems for poorly water-soluble drugs, Molecules 20 (2015) 20397-20408. 
[49] A. Varela-Moreira, Y. Shi, M.H. Fens, T. Lammers, W.E. Hennink, R.M. Schiffelers, Clinical application of polymeric micelles for the treatment of cancer, Materials Chemistry Frontiers 1 (2017) 1485-1501.

[50] X. Ju, L. Chu, Lab-on-a-chip fabrication of polymeric microparticles for drug encapsulation and controlled release, In: Micro and Nano Technologies, Microfluidics for Pharmaceutical Applications, Editors: H.A. Santos, D. Liu, H. Zhang, William Andrew Publishing, Norwich, NY, USA (2019).

[51] P. Kundu, S. Maiti, Cetyl gellan copolymer micelles and hydrogels: in vitro and pharmacodynamic assessment for drug delivery, International Journal of Biological Macromolecules 72 (2015) 1027-1033.

[52] S. Yan, J. Ren, Y. Jian, W. Wang, W. Yun, J. Yin, Injectable maltodextrin-based micelle/hydrogel composites for simvastatin-controlled release, Biomacromolecules 19 (2018) $4554-4564$

[53] M. Chen, H. Tsai, C. Liu, S. Peng, W. Lai, S. Chen, Y. Chang, H. Sung, A nanoscale drugentrapment strategy for hydrogel-based systems for the delivery of poorly soluble drugs, Biomaterials 30 (2009) 2102-2111.

[54] A.M. Zhu, J. Hua Chen, Q.L. Liu, Y.L. Jiang, Controlled release of berberine hydrochloride from alginate microspheres embedded within carboxymethyl chitosan hydrogels, Journal of Applied Polymer Science 120 (2011) 2374-2380.

[55] Z. Cong, Y. Shi, Y. Wang, Y. Wang, J. Niu, N. Chen, H. Xue, A novel controlled drug delivery system based on alginate hydrogel/chitosan micelle composites, International Journal of Biological Macromolecules 107 (2018) 855-864.

[56] H. Cabral, K. Kataoka, Progress of drug-loaded polymeric micelles into clinical studies, Journal of Controlled Release 190 (2014) 465-476. 
[57] J.L. Markman, A. Rekechenetskiy, E. Holler, J.Y. Ljubimova, Nanomedicine therapeutic approaches to overcome cancer drug resistance, Advanced Drug Delivery Reviews 65 (2013) 1866-1879.

[58] D. Zhong, Z. Liu, S. Xie, W. Zhang, Y. Zhang, W. Xue, Study on poly(D, L-lactic) microspheres embedded in calcium alginate hydrogel beads as dual drug delivery systems, Journal of Applied Polymer Science 129 (2013) 767-772.

[59] R.M. Rasal, A.V. Janorkar, D.E. Hirt, Poly(lactic acid) modifications, Progress in Polymer Science 35 (2010) 338-356.

[60] E. Ruel-Gariépy, J. Leroux, In situ-forming hydrogels - review of temperature-sensitive systems, European Journal of Pharmaceutics and Biopharmaceutics 58 (2004) 409-426.

[61] S.R. Van Tomme, G. Storm, W.E. Hennink, In situ gelling hydrogels for pharmaceutical and biomedical applications, International Journal of Pharmaceutics 355 (2008) 1-18.

[62] X. Chen, M. Fan, H. Tan, B. Ren, G. Yuan, Y. Jia, J. Li, D. Xiong, X. Xing, X. Niu, X. $\mathrm{Hu}$, Magnetic and self-healing chitosan-alginate hydrogel encapsulated gelatin microspheres via covalent cross-linking for drug delivery, Materials Science and Engineering: C 101 (2019) 619-629.

[63] M. Fan, Y. Ma, H. Tan, Y. Jia, S. Zou, S. Guo, M. Zhao, H. Huang, Z. Ling, Y. Chen, X. $\mathrm{Hu}$, Covalent and injectable chitosan-chondroitin sulfate hydrogels embedded with chitosan microspheres for drug delivery and tissue engineering, Materials Science and Engineering: C 71 (2017) 67-74.

[64] R. Langer, J. Vacanti, Tissue engineering, Science 260 (1993), 920-926.

[65] X. Jia, K.L. Kiick, Hybrid multicomponent hydrogels for tissue engineering, Macromolecular Bioscience 9 (2009) 140-156.

[66] J.M. Shapiro, M.L. Oyen, Hydrogel composite materials for tissue engineering scaffolds, JOM 65 (2013) 505-516. 
[67] J. Malda, J. Visser, F.P. Melchels, T. Jüngst, W.E. Hennink, W.J. Dhert, J. Groll, D.W. Hutmacher, 25th anniversary article: engineering hydrogels for biofabrication, Advanced Materials 25 (2013) 5011-5028.

[68] J. Zhang, B.J. Allardyce, R. Rajkhowa, Y. Zhao, R.J. Dilley, S.L. Redmond, X. Wang, X. Liu, 3D printing of silk particle-reinforced chitosan hydrogel structures and their properties, ACS Biomaterials Science \& Engineering 4 (2018) 3036-3046.

[69] M.T. Poldervaart, H. Wang, J. van der Stok, H. Weinans, S.C. Leeuwenburgh, F.C. Öner, W.J. Dhert, J. Alblas, Sustained release of BMP-2 in bioprinted alginate for osteogenicity in mice and rats, PLoS One 8 (2013) e72610.

[70] S. Xu, L. Deng, J. Zhang, L. Yin, A. Dong, Composites of electrospun-fibers and hydrogels: a potential solution to current challenges in biological and biomedical field, Journal of Biomedical Materials Research Part B 104 (2016) 640-656.

[71] L.A. Bosworth, L. Turner, S.H. Cartmell, State of the art composites comprising electrospun fibres coupled with hydrogels: a review, Nanomedicine: Nanotechnology, Biology and Medicine 9 (2013) 322-335.

[72] A.K. Means, M.A. Grunlan, Modern strategies to achieve tissue-mimetic, mechanically robust hydrogels, ACS Macro Letters 8 (2019) 705-713.

[73] S. Khorshidi, A. Karkhaneh, A review on gradient hydrogel/fiber scaffolds for osteochondral regeneration, Journal of Tissue Engineering and Regenerative Medicine 12 (2018) e1974-e1990.

[74] J.P. Maranchi, M.M. Trexler, Q. Guo, J.H. Elisseeff, Fibre-reinforced hydrogels with high optical transparency, International Materials Reviews 59 (2014) 264-296.

[75] K. Tonsomboon, A.L. Butcher, M.L. Oyen, Strong and tough nanofibrous hydrogel composites based on biomimetic principles, Materials Science and Engineering: C 72 (2017) 220-227. 
[76] K. Tonsomboon, M.L. Oyen, Composite electrospun gelatin fiber-alginate gel scaffolds for mechanically robust tissue engineered cornea, Journal of the Mechanical Behavior of Biomedical Materials 21 (2013) 185-194.

[77] L.D. Wright, K. McKeon-Fischer, Z. Cui, L.S. Nair, J.W. Freeman, PDLA/PLLA and PDLA/PCL nanofibers with a chitosan-based hydrogel in composite scaffolds for tissue engineered cartilage, Journal of Tissue Engineering and Regenerative Medicine 8 (2014) 946954.

[78] F. Mohabatpour, A. Karkhaneh, A.M. Sharifi, A hydrogel/fiber composite scaffold for chondrocyte encapsulation in cartilage tissue regeneration, RSC Advances 6 (2016) 8313583145.

[79] D. Benayahu, M. Sharabi, L. Pomeraniec, L. Awad, R. Haj-Ali, Y. Benayahu, Unique collagen fibers for biomedical applications, Marine Drugs 16 (2018) 102.

[80] S. Poveda-Reyes, L.R. Mellera-Oglialoro, R. Martínez-Haya, T.C. Gamboa-Martínez, J.L. Gomez Ribelles, G. Gallego Ferrer, Reinforcing an injectable gelatin hydrogel with PLLA microfibers: two routes for short fiber production, Macromolecular Materials and Engineering 300 (2015) 977-988.

[81] F. Mirahmadi, M. Tafazzoli-Shadpour, M.A. Shokrgozar, S. Bonakdar, Enhanced mechanical properties of thermosensitive chitosan hydrogel by silk fibers for cartilage tissue engineering, Materials Science and Engineering: C 33 (2013) 4786-4794.

[82] O. Regev, C.S. Reddy, N. Nseir, E. Zussman, Hydrogel reinforced by short albumin fibers: mechanical characterization and assessment of biocompatibility, Macromolecular Materials and Engineering 298 (2013) 283-291.

[83] Y. Yang, I. Wimpenny, M. Ahearne, Portable nanofiber meshes dictate cell orientation throughout three-dimensional hydrogels, Nanomedicine: Nanotechnology, Biology and Medicine 7 (2011) 131-136. 
[84] A. Hsieh, T. Zahir, Y. Lapitsky, B. Amsden, W. Wan, M.S. Shoichet, Hydrogel/electrospun fiber composites influence neural stem/progenitor cell fate, Soft Matter 6 (2010) 2227-2237.

[85] O. Pinkas, O. Haneman, O. Chemke, M. Zilberman, Fiber-reinforced composite hydrogels for bioadhesive and sealant applications, Polymers for Advanced Technologies 28 (2017) 11621169.

[86] O. Pinkas, M. Zilberman, Effect of hemostatic agents on properties of gelatin-alginate soft tissue adhesives, Journal of Biomaterials Science, Polymer Edition 25 (2014) 555-573.

[87] B. Cohen, O. Pinkas, M. Foox, M. Zilberman, Gelatin-alginate novel tissue adhesives and their formulation-strength effects, Acta Biomaterialia 9 (2013) 9004-9011.

[88] N. Han, J. Johnson, J.J. Lannutti, J.O. Winter, Hydrogel-electrospun fiber composite materials for hydrophilic protein release, Journal of Controlled Release 158 (2012) 165-170.

[89] R. Elia, D.R. Newhide, P.D. Pedevillano, G.R. Reiss, M.A. Firpo, E.W. Hsu, D.L. Kaplan, G.D. Prestwich, R.A. Peattie, Silk-hyaluronan-based composite hydrogels: a novel, securable vehicle for drug delivery, Journal of Biomaterials Applications 27 (2013) 749-762.

[90] H.P.S. Abdul Khalil, A.H. Bhat, A.F. Ireana Yusra, Green composites from sustainable cellulose nanofibrils: a review, Carbohydrate Polymers 87 (2012) 963-979.

[91] H.P.S. Abdul Khalil, C.K. Saurabha, A.S. Adnan, M.R. Nurul Fazita, M.I. Syakir, Y. Davoudpour, M. Rafatullah, C.K. Abdullah, M.K.M. Haafiz, R. Dungani, A review on chitosancellulose blends and nanocellulose reinforced chitosan biocomposites: properties and their applications, Carbohydrate Polymers 150 (2016) 216-226.

[92] A.J. Onyianta, M. Castellano, M. Dorris, R.L. Williams, S. Vicini, The effects of morpholine pre-treated and carboxymethylated cellulose nanofibrils on the properties of alginate-based hydrogels, Carbohydrate Polymers 198 (2018) 320-327. 
[93] R. Jonas, L.F. Farah, Production and application of microbial cellulose, Polymer Degradation and Stability 59 (1998) 101-106.

[94] K.J. De France, T. Hoare, E.D. Cranston, Review of hydrogels and aerogels containing nanocellulose, Chemistry of Materials 29 (2017) 4609-4631.

[95] D.R. Pereira, J. Silva-Correia, J.M. Oliveira, R.L. Reis, A. Pandit, M.J. Biggs, Nanocellulose reinforced gellan-gum hydrogels as potential biological substitutes for annulus fibrosus tissue regeneration, Nanomedicine: Nanotechnology, Biology and Medicine 14 (2018) 897-908.

[96] I. Doench, T.A. Tran, L. David, A. Montembault, E. Viguier, C. Gorzelanny, G. Sudre, T. Cachon, M. Louback-Mohamed, N. Horbelt, Cellulose nanofiber-reinforced chitosan hydrogel composites for intervertebral disc tissue repair, Biomimetics 4 (2019) 19.

[97] U.T.M. Sampath, Y.C. Ching, C.H. Chuah, R. Singh, P. Lin, Preparation and characterization of nanocellulose reinforced semi-interpenetrating polymer network of chitosan hydrogel, Cellulose 24 (2017) 2215-2228.

[98] S.A.A. Ghavimi, E.S. Lungren, T.J. Faulkner, M.A. Josselet, Y. Wu, Y. Sun, F.M. Pfeiffer, C.L. Goldstein, C. Wan, B.D. Ulery, Inductive co-crosslinking of cellulose nanocrystal/chitosan hydrogels for the treatment of vertebral compression fractures, International Journal of Biological Macromolecules 130 (2019) 88-98.

[99] M.R. Hossen, N. Dadoo, D.G. Holomakoff, A. Co, W.M. Gramlich, M.D. Mason, Wet stable and mechanically robust cellulose nanofibrils (CNF) based hydrogel, Polymer 151 (2018) 231-241.

[100] S. Sultan, A.P. Mathew, 3D printed scaffolds with gradient porosity based on a cellulose nanocrystal hydrogel, Nanoscale 10 (2018) 4421-4431. 
[101] R.E. Abouzeid, R. Khiari, D. Beneventi, A. Dufresne, Biomimetic mineralization of three-dimensional printed alginate/TEMPO-oxidized cellulose nanofibril scaffolds for bone tissue engineering, Biomacromolecules 19 (2018) 4442-4452.

[102] H. Martínez Ávila, S. Schwarz, N. Rotter, P. Gatenholm, 3D bioprinting of human chondrocyte-laden nanocellulose hydrogels for patient-specific auricular cartilage regeneration, Bioprinting 1-2 (2016) 22-35.

[103] K. Markstedt, A. Mantas, I. Tournier, H. Martínez Ávila, D. Hägg, P. Gatenholm, 3D bioprinting human chondrocytes with nanocellulose-alginate bioink for cartilage tissue engineering applications, Biomacromolecules 16 (2015) 1489-1496.

[104] D. Nguyen, D.A. Hägg, A. Forsman, J. Ekholm, P. Nimkingratana, C. Brantsing, T. Kalogeropoulos, S. Zaunz, S. Concaro, M. Brittberg, Cartilage tissue engineering by the 3D bioprinting of iPS cells in a nanocellulose/alginate bioink, Scientific Reports 7 (2017) 658.

[105] Q. Xu, Y. Ji, Q. Sun, Y. Fu, Y. Xu, L. Jin, Fabrication of cellulose nanocrystal/chitosan hydrogel for controlled drug release, Nanomaterials 9 (2019) 253.

[106] X. Zheng, Q. Zhang, J. Liu, Y. Pei, K. Tang, A unique high mechanical strength dialdehyde microfibrillated cellulose/gelatin composite hydrogel with a giant network structure, RSC Advances 6 (2016) 71999-72007.

[107] M. Park, D. Lee, J. Hyun, Nanocellulose-alginate hydrogel for cell encapsulation, Carbohydrate Polymers 116 (2015) 223-228.

[108] H. Yan, D. Huang, X. Chen, H. Liu, Y. Feng, Z. Zhao, Z. Dai, X. Zhang, Q. Lin, A novel and homogeneous scaffold material: preparation and evaluation of alginate/bacterial cellulose nanocrystals/collagen composite hydrogel for tissue engineering, Polymer Bulletin 75 (2018) 985-1000. 
[109] Y. Jia, X. Wang, M. Huo, X. Zhai, F. Li, C. Zhong, Preparation and characterization of a novel bacterial cellulose/chitosan bio-hydrogel, Nanomaterials and Nanotechnology 7 (2017) 1847980417707172.

[110] P. Zhang, L. Chen, Q. Zhang, F.F. Hong, Using in situ dynamic cultures to rapidly biofabricate fabric-reinforced composites of chitosan/bacterial nanocellulose for antibacterial wound dressings, Frontiers in Microbiology 7 (2016) 260. 